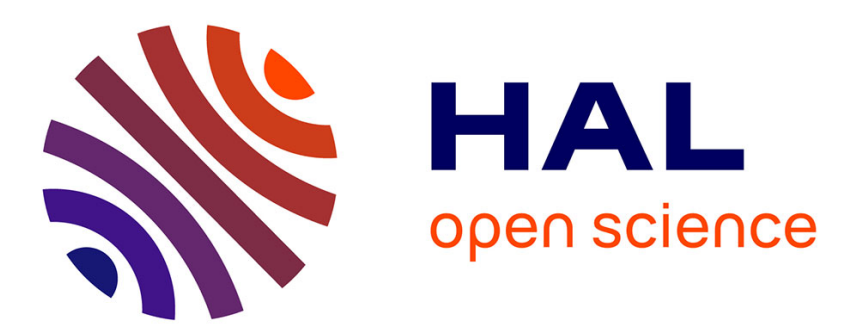

\title{
Null controllability via comparison results for nonlinear age-structured population dynamics
}

Sebastian Anita, Nicolas Hegoburu

\section{To cite this version:}

Sebastian Anita, Nicolas Hegoburu. Null controllability via comparison results for nonlinear agestructured population dynamics. Mathematics of Control, Signals, and Systems, 2019, 31:2. hal01861757

\section{HAL Id: hal-01861757 \\ https://hal.science/hal-01861757}

Submitted on 25 Aug 2018

HAL is a multi-disciplinary open access archive for the deposit and dissemination of scientific research documents, whether they are published or not. The documents may come from teaching and research institutions in France or abroad, or from public or private research centers.
L'archive ouverte pluridisciplinaire HAL, est destinée au dépôt et à la diffusion de documents scientifiques de niveau recherche, publiés ou non, émanant des établissements d'enseignement et de recherche français ou étrangers, des laboratoires publics ou privés. 


\title{
Null controllability via comparison results for nonlinear age-structured population dynamics
}

\author{
Sebastian Aniţa*, Nicolas Hegoburu ${ }^{\dagger}$
}

August 25, 2018

\begin{abstract}
We consider an infinite dimensional nonlinear controlled system describing age-structured population dynamics, where the birth and the mortality rates are nonlinear functions of the population size. The control being active on some age range, we give sharp conditions subject to the age range and the control time horizon to get the null controllability of the nonlinear controlled population dynamics. The main novelty is that we use here as a main ingredient the comparison principle for age-structured population dynamics, and in case of null controllability we provide a feedback control with a very simple structure, while preserving the nonnegativity of the state trajectory. Finally, we establish the lack of the null controllability for the linear Lotka-McKendrick equation with spatial diffusion when the control acts in a subset of the habitat and we want to preserve the positivity of the state trajectory.
\end{abstract}

\section{Introduction}

We shall continue and extend the investigation in Hegoburu, Magal and Tucsnak [8] concerning the null controllability of the age-dependent population dynamics. More precisely, let $p(t, a)$ be the distribution of individuals of age $a$ at time $t$ of a biological population. Let $a_{\dagger}$ be the maximal age of an individual and $\tau$ be a positive constant. Consider that the population dynamics is described by the following nonlinear system, firstly proposed (without control) by Gurtin and MacCamy [7]:

$$
\left\{\begin{array}{lr}
\frac{\partial p}{\partial t}(t, a)+\frac{\partial p}{\partial a}(t, a)+\mu(t, a, P(t)) p(t, a)=\chi_{\left[a_{1}, a_{2}\right]}(a) u(t, a), & (t, a) \in Q_{\tau} \\
p(t, 0)=\int_{0}^{a_{\dagger}} \beta(t, a, P(t)) p(t, a) \mathrm{d} a, & t \in(0, \tau) \\
p(0, a)=p_{0}(a), & a \in\left(0, a_{\dagger}\right) \\
P(t)=\int_{0}^{a_{\dagger}} p(t, a) \mathrm{d} a, & t \in(0, \tau),
\end{array}\right.
$$

where $u$ is a control function, $\chi_{\left[a_{1}, a_{2}\right]}$ is the characteristic function of the interval $\left[a_{1}, a_{2}\right]$ (where $\left.0 \leqslant a_{1}<a_{2} \leqslant a_{\dagger}\right), p_{0}$ is the initial population density and $Q_{\tau}=(0, \tau) \times\left(0, a_{\dagger}\right) . \beta$ and $\mu$ are the fertility rate and the mortality rate, respectively. In the following, the nonlocal boundary condition

$$
p(t, 0)=\int_{0}^{a_{\dagger}} \beta(t, a, P(t)) p(t, a) \mathrm{d} a, \quad t \in(0, \tau),
$$

will be refered as the renewal law.

To state our main results, we first recall the standard assumptions, used for instance in Aniţa $\left[5\right.$, p. 30], on the functions $\mu, \beta, p_{0}$ and $u$ :

\footnotetext{
*Faculty of Mathematics, "Alexandru Ioan Cuza" University of Iaşi, and "Octav Mayer" Institute of Mathematics of the Romanian Academy, Iaşi 700506, Romania, E-mail: sanita@uaic.ro

${ }^{\dagger}$ Institut de Mathématiques de Bordeaux, Université de Bordeaux/Bordeaux INP/CNRS, 351 Cours de la Libération, 33405 TALENCE, France, E-mail: nicolas.hegoburu@math.u-bordeaux.fr
} 
(H1) for every $s \geqslant 0$, the functions $\beta(\cdot, \cdot, s)$ and $\mu(\cdot, \cdot, s)$ belong to $L^{\infty}\left(Q_{\tau}\right)$ and $L_{l o c}^{1}\left([0, \tau] \times\left[0, a_{\dagger}\right)\right)$ respectively,

(H2) the functions $\beta$ and $\mu$ are locally Lipschitz functions with respect to the third variable, i.e. for any $M>0$, there exists $L(M)>0$ such that for almost every $(t, a) \in Q_{\tau}$ and for every $s_{1}, s_{2} \in[0, M]$, we have

$$
\begin{aligned}
& \left|\beta\left(t, a, s_{1}\right)-\beta\left(t, a, s_{2}\right)\right| \leqslant L(M) \cdot\left|s_{1}-s_{2}\right|, \\
& \left|\mu\left(t, a, s_{1}\right)-\mu\left(t, a, s_{2}\right)\right| \leqslant L(M) \cdot\left|s_{1}-s_{2}\right|,
\end{aligned}
$$

(H3) for almost every $(t, a, s) \in Q_{\tau} \times(0,+\infty)$, we have

$$
\beta(t, a, s) \geqslant 0 \quad \text { and } \quad \mu(t, a, s) \geqslant 0,
$$

(H4) for almost every $(t, a) \in Q_{\tau}$, the function $\beta(t, a, \cdot)$ is nonincreasing,

(H5) for almost every $(t, a) \in Q_{\tau}$, the function $\mu(t, a, \cdot)$ is nondecreasing,

(H6) $p_{0} \in L^{2}\left(0, a_{\dagger}\right), p_{0}(a) \geqslant 0$ a.e. $a \in\left(0, a_{\dagger}\right)$,

(H7) the control function $u$ belongs to $L^{2}\left(Q_{\tau}\right)$.

By a solution to (1.1), we mean a function $p \in L^{\infty}\left(0, \tau ; L^{2}\left(0, a_{\dagger}\right)\right)$, absolutely continuous along almost every characteristic line of equation $t-a=c$ (with $c \in\left(-a_{\dagger}, \tau\right)$ ), which satisfies

$$
\left\{\begin{array}{lr}
D p(t, a)+\mu(t, a, P(t)) p(t, a)=\chi_{\left[a_{1}, a_{2}\right]}(a) u(t, a) & \text { a.e. }(t, a) \in Q_{\tau} \\
\lim _{\varepsilon \rightarrow 0^{+}} p(t+\varepsilon, \varepsilon)=\int_{0}^{a_{\dagger}} \beta(t, a, P(t)) p(t, a) \mathrm{d} a & \text { a.e. } t \in(0, \tau) \\
\lim _{\varepsilon \rightarrow 0^{+}} p(\varepsilon, a+\varepsilon)=p_{0}(a) & \text { a.e. } a \in\left(0, a_{\dagger}\right),
\end{array}\right.
$$

where $P(t)=\int_{0}^{a_{\dagger}} p(t, a) \mathrm{d} a$ for almost every $t \in(0, \tau)$. Here

$$
D p(t, a)=\lim _{\varepsilon \rightarrow 0} \frac{p(t+\varepsilon, a+\varepsilon)-p(t, a)}{\varepsilon}
$$

is the directional derivative of $p$ at $(t, a)$ for direction $(1,1)$. The definition of the solution implies that there is a trace of $p$ on any curve of equation $t=c$, with $c \in[0, \tau]$. With the above assumptions system (1.1) has at most one nonnegative solution, and if in addition $u(t, a) \geq 0$ a.e. $(t, a) \in Q_{\tau}$, the existence and uniqueness of a nonnegative solution to (1.1) can be proved via the Banach fixed point theorem (see, for instance, [5, Chapter 2]).

Notice that actually the control acts only for $a \in\left[a_{1}, a_{2}\right]$.

For almost every $(t, a) \in Q_{\tau}$, denote by $\beta_{+}(t, a):=\beta(t, a, 0)$ so that, due to assumption (H4), for almost every $(t, a, s) \in Q_{\tau} \times(0,+\infty)$ we have

$$
\beta(t, a, s) \leqslant \beta(t, a, 0)=\beta_{+}(t, a) \quad \text { a.e. }(t, a, s) \in Q_{\tau} \times(0,+\infty) .
$$

Our first result asserts that, assuming that the age of individuals able to reproduce is bounded away from zero, given $a_{1}$ small enough and $\tau$ large enough, we are able to bring the solution $p$ of (1.1) to zero by means of a control function $u \in L^{2}\left(Q_{\tau}\right)$, preserving the nonnegativity of the state trajectory. More precisely, we have 
Theorem 1.1. With the above notations and assumptions, suppose that there exists $a_{b} \in\left(0, a_{\dagger}\right)$ such that for almost every $t \in(0, \tau)$, we have

$$
\beta_{+}(t, a)=0 \quad \text { a.e. } a \in\left(0, a_{b}\right),
$$

where the function $\beta_{+}$is defined in (1.2).

Assume that we have $a_{1}<a_{b}$. If $\tau>a_{\dagger}-a_{2}+a_{1}$, then for every $p_{0} \in L^{2}\left(0, a_{\dagger}\right)$ with $p_{0}(a) \geqslant 0$ a.e. $a \in\left(0, a_{\dagger}\right)$, there exists $u \in L^{2}\left(Q_{\tau}\right)$ and a corresponding unique nonnegative solution $p$ of (1.1) such that

$$
p(\tau, a)=0 \quad \text { a.e. } a \in\left(0, a_{\dagger}\right) .
$$

The following Theorem 1.2 states that the previous result is sharp, in the sense that if $a_{1}$ (respectively $\tau$ ) is bigger or equal than $a_{b}$ (respectively smaller than $a_{\dagger}-a_{2}+a_{1}$ ), the system (1.1) may not be null controllable by means of controls $u \in L^{2}\left(Q_{\tau}\right)$ while preserving the nonnegativity of the controlled state trajectory. More precisely, we have

Theorem 1.2. With the above notations and assumptions,

(i) Assume that there exists $a_{b} \in\left(0, a_{\dagger}\right)$ and $a_{m} \in\left(0, a_{\dagger}\right]$ with $a_{b}<a_{m}$ such that, for almost every $t \in(0, \tau)$ we have

$$
\beta(t, a, s)>0 \quad \text { a.e. }(a, s) \in\left(a_{b}, a_{m}\right) \times(0,+\infty) .
$$

If $a_{1} \geqslant a_{b}$, then for every $\tau>0$, there exists $p_{0} \in L^{2}\left(0, a_{\dagger}\right)$ with $p_{0}(a) \geqslant 0$ a.e. $a \in\left(0, a_{\dagger}\right)$ such that there is no control $u$ and a corresponding nonnegative solution $p$ to (1.1) satisfying

$$
p(\tau, a)=0 \quad \text { a.e. } a \in\left(0, a_{\dagger}\right) .
$$

(ii) If $\tau<a_{\dagger}-a_{2}+a_{1}$, there exists a function $\beta$ satisfying (H1) - (H4) and an initial population density $p_{0} \in L^{2}\left(0, a_{\dagger}\right)$ with $p_{0}(a) \geqslant 0$ a.e. $a \in\left(0, a_{\dagger}\right)$ such that there is no control $u$ and $a$ corresponding nonnegative solution $p$ to (1.1) satisfying

$$
p(\tau, a)=0 \quad \text { a.e. } a \in\left(0, a_{\dagger}\right) .
$$

Our third result asserts that condition (1.3) is not necessary to get the null controllability of system (1.1), provided that we are able to control the very young individuals. More precisely, provided that $a_{1}=0$ and $a_{2}>0$, for any function $\beta$ satisfying $(\mathbf{H} 1)-(\mathbf{H} 4)$, we are able to bring the solution $p$ of (1.1) to zero in sufficiently large time $\tau$ by means of a control $u \in L^{2}\left(Q_{\tau}\right)$, preserving the nonnegativity of the state trajectory:

Theorem 1.3. With the above notations and assumptions,

(i) Assume that $a_{1}=0$ and $a_{2}>0$. If $\tau>a_{\dagger}-a_{2}$, then for every $p_{0} \in L^{2}\left(0, a_{\dagger}\right)$ with $p_{0}(a) \geqslant 0$ a.e. $a \in\left(0, a_{\dagger}\right)$, there exists $u \in L^{2}\left(Q_{\tau}\right)$ and a unique nonnegative solution $p$ of (1.1) such that

$$
p(\tau, a)=0 \quad \text { a.e. } a \in\left(0, a_{\dagger}\right) .
$$

(ii) Assume that there exists $a_{m} \in\left(0, a_{\dagger}\right]$ such that, for almost every $t \in(0, \tau)$ we have

$$
\beta(t, a, s)>0 \quad \text { a.e. }(a, s) \in\left(0, a_{m}\right) \times(0,+\infty) .
$$

If $a_{1}>0$, then for every $\tau>0$, there exists $p_{0} \in L^{2}\left(0, a_{\dagger}\right)$ with $p_{0}(a) \geqslant 0$ a.e. $a \in\left(0, a_{\dagger}\right)$ such that there is no control $u$ and a corresponding nonnegative solution $p$ to (1.1) satisfying

$$
p(\tau, a)=0 \quad \text { a.e. } a \in\left(0, a_{\dagger}\right) .
$$


(iii) If $\tau<a_{\dagger}-a_{2}$, there exists an initial population density $p_{0} \in L^{2}\left(0, a_{\dagger}\right)$ with $p_{0}(a) \geqslant 0$ a.e. $a \in\left(0, a_{\dagger}\right)$ such that there is no control $u$ and a corresponding nonnegative solution $p$ to (1.1) satisfying

$$
p(\tau, a)=0 \quad \text { a.e. } a \in\left(0, a_{\dagger}\right) .
$$

For an overview on age-structured population dynamics models we refer, for instance, to Webb [19], Iannelli [10], Kunisch et al [13] and the references therein.

In the case where both functions $\beta$ and $\mu$ depend only on the age variable, some null controllability results of the age-dependent population dynamics model (without diffusion) were first obtained by Barbu, Iannelli and Martcheva [6]. Assuming that the control is supported in the age interval $\left(0, a_{0}\right)$, for some $a_{0}<a_{\dagger}$, the authors proved that the controlled population may be steered to any steady state of (1.1), except for a small interval of ages near zero. Recently, Hegoburu, Magal and Tucsnak [8] proved that this restriction is not necessary, provided individuals do not reproduce at the age close to zero. They also proved there exists controls which preserves the nonnegativity of the state trajectory. More recently, Maity [14] proved that null controllability can be achieved by controls supported in any subinterval $\left[a_{1}, a_{2}\right]$ of $\left[0, a_{\dagger}\right]$, provided we control before the individuals start to reproduce. In this case, the author proved that the system is null controllable in sufficiently large time, i.e. when $\tau>a_{1}+\max \left\{a_{1}, a_{\dagger}-a_{2}\right\}$ by means of observability inequalities.

The null controllability of systems modelling nonlinear age-structured population dynamics has been studied by Ainseba and Iannelli [3], in the case when $a_{1}=0$ and $a_{2} \in\left(0, a_{\dagger}\right)$, using as a main ingredient the Kakutani fixed-point theorem. In [3] the main result asserts controllability of the system (1.1) to zero in time $\tau>a_{\dagger}$ by a distributed control, except for a small interval of ages near zero. In our present paper, we get an exact null controllability result for the system (1.1). Moreover, the minimal time needed to steer the population to zero (i.e. $\tau>a_{\dagger}-a_{2}+a_{1}$ ) seems to be sharp. Instead of using observability results we use here as a main ingredient the comparison principle for linear age-structured population dynamics. In case of null controllability we provide a feedback control with a very simple structure and such that (1.1) admits a unique nonnegative solution $p$ satisfying $p(\tau, a)=0$ a.e. $a \in\left(0, a_{\dagger}\right)$.

As already mentionned, in the present work we use comparison results for age-structured population dynamics and some feedback controls with a simple structure (as a harvesting term) in order to prove some null-controllability results. Actually, the use of such harvesting term in order to get the null-controllability in an abstract space is presented in Section 2. Sections 3, 4 and 5 are devoted to prove respectively Theorems 1.1, 1.2 and 1.3 relatively to the linear setting, i.e. the above mentioned Theorems will be proved in the case where the functions $\beta$ and $\mu$ do not depend on the third variable. In Section 6 we give two applications to the results obtained in the three previous Sections: we first show how to steer a population to another one in the linear setting, preserving the nonnegativity of the population, and we prove Theorems 1.1, 1.2 and 1.3 without restriction on the functions $\beta$ and $\mu$ (i.e. in the general nonlinear case) using as a main ingredient the comparison principle for age-structured populations dynamics. In Section 7, we show how the comparison principle for age-structured population dynamics may be used to prove the lack of the null controllability for the linear Lotka-McKendrick equation with spatial diffusion when the control acts in a subset of the habitat.

\section{A null-controllability result in an abstract space}

Consider the following controlled system:

$$
\left\{\begin{array}{l}
y^{\prime}(t)-A y(t)=u(t), \quad t \in(0, \tau) \\
y(0)=y_{0}
\end{array}\right.
$$

where $A$ is the generator of a $\mathcal{C}_{0}$ - semigroup $\left(e^{t A}\right)_{t \geq 0}$ of linear and bounded operators in the real Banach space $X$ and $\tau$ is a positive constant. We remind that by a mild solution to (2.1), we mean 
the function $y \in \mathcal{C}([0, \tau] ; X)$ given by

$$
y(t)=e^{t A} y_{0}+\int_{0}^{t} e^{(t-s) A} u(s) \mathrm{d} s, \quad \text { for every } t \in[0, \tau] .
$$

If $y_{0} \in X$ and $u \in L^{2}(0, \tau ; X)$, then (2.1) has a unique mild solution.

The controllability properties of (2.1) have been investigated by Tucsnak and Weiss [18] via the observability. Here we use a different approach in order to get the null-controllability: we use a feedback control. Actually, if

$$
u(t):=-\frac{1}{\tau-t} y(t), \quad t \in(0, \tau)
$$

then (2.1) becomes

$$
\left\{\begin{array}{l}
y^{\prime}(t)-A y(t)=-\frac{1}{\tau-t} y(t), \quad t \in(0, \tau) \\
y(0)=y_{0},
\end{array}\right.
$$

and by a mild solution to $(2.3)$ we mean a function $y \in \mathcal{C}([0, \tau] ; X)$ such that

$$
y(t)=e^{t A} y_{0}-\int_{0}^{t} e^{(t-s) A}\left(\frac{1}{\tau-s} y(s)\right) \mathrm{d} s, \quad \text { for every } t \in[0, \tau] .
$$

By Gronwall-Bellman's inequality we may conclude that there exists at most one mild solution to (2.3). Let us prove that actually, the function $y$ defined by

$$
y(t):=\frac{\tau-t}{\tau} e^{t A} y_{0} \quad t \in[0, \tau],
$$

is a mild solution to $(2.3)$ and that $u$ given by $(2.2)$ belongs to $L^{2}(0, \tau ; X)$.

Indeed, $y$ given by $(2.4)$ belongs to $\mathcal{C}([0, \tau] ; X)$ and for any $t \in[0, \tau]$ we have

$$
\begin{aligned}
& e^{t A} y_{0}-\int_{0}^{t} \frac{1}{\tau-s} e^{(t-s) A} y(s) \mathrm{d} s \\
& =e^{t A} y_{0}-\frac{1}{\tau} \int_{0}^{t} \frac{1}{\tau-s}(\tau-s) e^{(t-s) A} e^{s A} y_{0} \mathrm{~d} s \\
& =e^{t A} y_{0}-\frac{t}{\tau} e^{t A} y_{0}=\frac{\tau-t}{\tau} e^{t A} y_{0}=y(t) .
\end{aligned}
$$

On the other hand, the control $u$ given by (2.2) satisfies

$$
u(t)=-\frac{1}{\tau-t} y(t)=-\frac{1}{\tau} e^{t A} y_{0}, \quad t \in[0, \tau],
$$

which indeed belongs to $C([0, \tau] ; X)$ and consequently to $L^{2}(0, \tau ; X)$.

Notice that, assuming that the functions $\beta$ and $\mu$ depend only on the age variable, the formulation of equations (1.1) may be considered using semigroup theory. Indeed, denote by $X=L^{2}\left(0, a_{\dagger}\right)$ and consider the operator $A: \mathcal{D}(A) \rightarrow X$ defined by

$$
\begin{aligned}
& \mathcal{D}(A)=\left\{\varphi \in L^{2}\left(0, a_{\dagger}\right) ; \varphi(0)=\int_{0}^{a_{\dagger}} \beta(a) \varphi(a) \mathrm{d} a,-\frac{\mathrm{d} \varphi}{\mathrm{d} a}-\mu \varphi \in L^{2}\left(0, a_{\dagger}\right)\right\}, \\
& A \varphi=-\frac{\mathrm{d} \varphi}{\mathrm{d} a}-\mu \varphi,
\end{aligned}
$$

It is well known (see, for instance, Song et al. [16] or Kappel and Zhang [11]) that $A$ generates a $\mathcal{C}_{0}$ - semigroup of linear and bounded operators in $X$ which we denote by $\left(e^{t A}\right)_{t \geqslant 0}$. If the control 
function $u$ in (1.1) acts in the whole age range $\left(0, a_{\dagger}\right)$ (i.e. if $a_{1}=0$ and $\left.a_{2}=a_{\dagger}\right)$ and is defined by the feedback form

$$
u(t):=-\frac{1}{\tau-t} p(t), \quad t \in[0, \tau],
$$

where $p$ is the corresponding solution to (1.1), we deduce that $p$ satisfies

$$
\left\{\begin{array}{lr}
\frac{\partial p}{\partial t}(t, a)+\frac{\partial p}{\partial a}(t, a)+\left(\mu(a)+\frac{1}{\tau-t}\right) p(t, a)=0, & (t, a) \in Q_{\tau} \\
p(t, 0)=\int_{0}^{a_{\dagger}} \beta(a) p(t, a) \mathrm{d} a, & t \in(0, \tau) \\
p(0, a)=p_{0}(a), & a \in\left(0, a_{\dagger}\right),
\end{array}\right.
$$

and so that for every $t \in[0, \tau]$ we get from (2.4) that $p(t)=\frac{\tau-t}{\tau} e^{t A} p_{0}$ in $X$ for any $t \in[0, \tau]$, and $p(\tau)=0$ in $X$. It can be seen in (2.6) that, $u$ given in such a feedback form (2.5), it behaves like an additional mortality (as a harvesting rate) for the age-structured population dynamics. In Sections 3 and 5 we shall develop this idea in the more general case when the functions $\beta$ and $\mu$ depend on both time and age variable, and the control $u$ acts in a subinterval of $\left[0, a_{\dagger}\right]$.

\section{The linear case : a positive null controllability result when the young individuals do not reproduce}

In this section, we shall prove Theorem 1.1 in the case where the functions $\beta$ and $\mu$ do not depend on the third variable. In other words, we assume that the population dynamics is described by the following linear system:

$$
\left\{\begin{array}{lr}
\frac{\partial p}{\partial t}(t, a)+\frac{\partial p}{\partial a}(t, a)+\mu_{0}(t, a) p(t, a)=\chi_{\left[a_{1}, a_{2}\right]}(a) u(t, a), & (t, a) \in Q_{\tau} \\
p(t, 0)=\int_{0}^{a_{\dagger}} \beta_{0}(t, a) p(t, a) \mathrm{d} a, & t \in(0, \tau) \\
p(0, a)=p_{0}(a), & a \in\left(0, a_{\dagger}\right),
\end{array}\right.
$$

where $\tau$ is a positive constant and $Q_{\tau}=(0, \tau) \times\left(0, a_{\dagger}\right)$. For the sake of completeness, let us recall the classical assumptions on the functions $\mu_{0}, \beta_{0}, p_{0}$ and $u$ relatively to the linear case:

(L1) the functions $\beta_{0}$ and $\mu_{0}$ belong to $L^{\infty}\left(Q_{\tau}\right)$ and $L_{l o c}^{1}\left([0, \tau] \times\left[0, a_{\dagger}\right)\right)$ respectively,

(L2) for almost every $(t, a) \in Q_{\tau}$, we have

$$
\beta_{0}(t, a) \geqslant 0 \text { and } \mu_{0}(t, a) \geqslant 0,
$$

(L3) $p_{0} \in L^{2}\left(0, a_{\dagger}\right), p_{0}(a) \geqslant 0$ a.e. $a \in\left(0, a_{\dagger}\right)$,

(L4) the control function $u$ belongs to $L^{2}\left(Q_{\tau}\right)$.

Note that, using the definition of a solution to (3.1), we can obtain, using an integration along the characteristic lines (see, for instance, [5, p. 16]) that for almost every $(t, a) \in Q_{\tau}$ with $a \geqslant t$, we have that the solution $p$ to (3.1) satistfies

$$
\begin{aligned}
& p(t, a)=\exp \left\{-\int_{0}^{t} \mu_{0}(s, a-t+s) \mathrm{d} s\right\} p_{0}(a-t) \\
& +\int_{0}^{t} \exp \left\{-\int_{s}^{t} \mu_{0}(\sigma, a-t+\sigma) \mathrm{d} \sigma\right\} \chi_{\left[a_{1}, a_{2}\right]}(a-t+s) u(s, a-t+s) \mathrm{d} s
\end{aligned}
$$


and for almost every $(t, a) \in Q_{\tau}$ with $t>a$, we have

$$
\begin{aligned}
& p(t, a)=\exp \left\{-\int_{0}^{a} \mu_{0}(t-a+s, s) \mathrm{d} s\right\} B(t-a) \\
& +\int_{0}^{a} \exp \left\{-\int_{s}^{a} \mu_{0}(t-a+\sigma, \sigma) \mathrm{d} \sigma\right\} \chi_{\left[a_{1}, a_{2}\right]}(s) u(t-a+s, s) \mathrm{d} s,
\end{aligned}
$$

where

$$
B(t)=\int_{0}^{a_{\dagger}} \beta_{0}(t, a) p(t, a) \mathrm{d} a \quad \text { a.e. } t \in(0, \tau) .
$$

Notice that, as a consequence of the Banach fixed-point theorem, we get that $B \in L^{\infty}(0, \tau)$ (see, for instance, [5]).

In this section, we will prove the following Proposition 3.1 which is, roughly speaking, the "linear version" of Theorem 1.1:

Proposition 3.1. With the above notations and assumptions, suppose that there exists $a_{b} \in\left(0, a_{\dagger}\right)$ such that for almost every $t \in(0, \tau)$, we have

$$
\beta_{0}(t, a)=0 \quad \text { a.e. } a \in\left(0, a_{b}\right) \text {. }
$$

Assume that we have $a_{1}<a_{b}$. If $\tau>a_{\dagger}-a_{2}+a_{1}$, then for every $p_{0} \in L^{2}\left(0, a_{\dagger}\right)$ with $p_{0}(a) \geqslant 0$ a.e. $a \in\left(0, a_{\dagger}\right)$, there exists $u \in L^{2}\left(Q_{\tau}\right)$ and a corresponding unique nonnegative solution $p$ of (3.1) such that

$$
p(\tau, a)=0 \quad \text { a.e. } a \in\left(0, a_{\dagger}\right) .
$$

In this whole section, we suppose that there exists $a_{b}>0$ such that (3.3) is satisfied, with $a_{1}<a_{b}$ and $\tau>a_{\dagger}-a_{2}+a_{1}$.

We may divide our proof in two cases, since the shapes of the controls functions driving the initial population to zero in time $\tau$ may be slightly different in the two following cases, depending on the order relationship of $a_{2}$ and $a_{b}$ :

Case $1: \tau>a_{\dagger}-a_{2}+a_{1}$ and $a_{1}<a_{2} \leqslant a_{b}$,

Case 2 : $\tau>a_{\dagger}-a_{2}+a_{1}$ and $a_{1}<a_{b}<a_{2}$.

The two following subsections are devoted to prove Proposition 3.1 in the two above mentioned cases.

\subsection{The first case : $\tau>a_{\dagger}-a_{2}+a_{1}$ and $a_{1}<a_{2} \leqslant a_{b}$}

This subsection is devoted to prove Proposition 3.1, in the case where $\tau>a_{\dagger}-a_{2}+a_{1}$ and $a_{1}<a_{2} \leqslant a_{b}$.

Proof of Proposition 3.1 with $a_{2} \leqslant a_{b}$. Suppose that $\tau>a_{\dagger}-a_{2}+a_{1}$ and $a_{1}<a_{2} \leqslant a_{b}$. Without loss of generality, we may assume that $\tau<a_{\dagger}$. Let us prove that there exists a feedback control $u$ and a corresponding unique nonnegative solution $p$ of (3.1) such that

$$
p(\tau, a)=0 \quad \text { a.e. } a \in\left(0, a_{\dagger}\right) .
$$

Denote by $\varepsilon:=\frac{1}{2}\left(\tau-\left(a_{\dagger}-a_{2}+a_{1}\right)\right)$. For almost every $(t, a) \in Q_{\tau}$, let us consider the feedback control $u(t, a):=-v(t, a) p(t, a)$, where $v$ is a control itself and acts as a harvesting rate, and is given by

$$
v(t, a):= \begin{cases}\frac{1}{a_{2}-a} & \text { if }\left\{\begin{array}{l}
\varepsilon-a_{2} \leqslant t-a<\tau-a_{2}, \\
a \in\left[a_{1}, a_{2}\right],
\end{array}\right. \\
\frac{1}{\tau-t} & \text { if }\left\{\begin{array}{l}
\tau-a_{2} \leqslant t-a \leqslant \tau-\left(a_{1}+\varepsilon\right), \\
a \in\left[a_{1}, a_{2}\right],
\end{array}\right.\end{cases}
$$




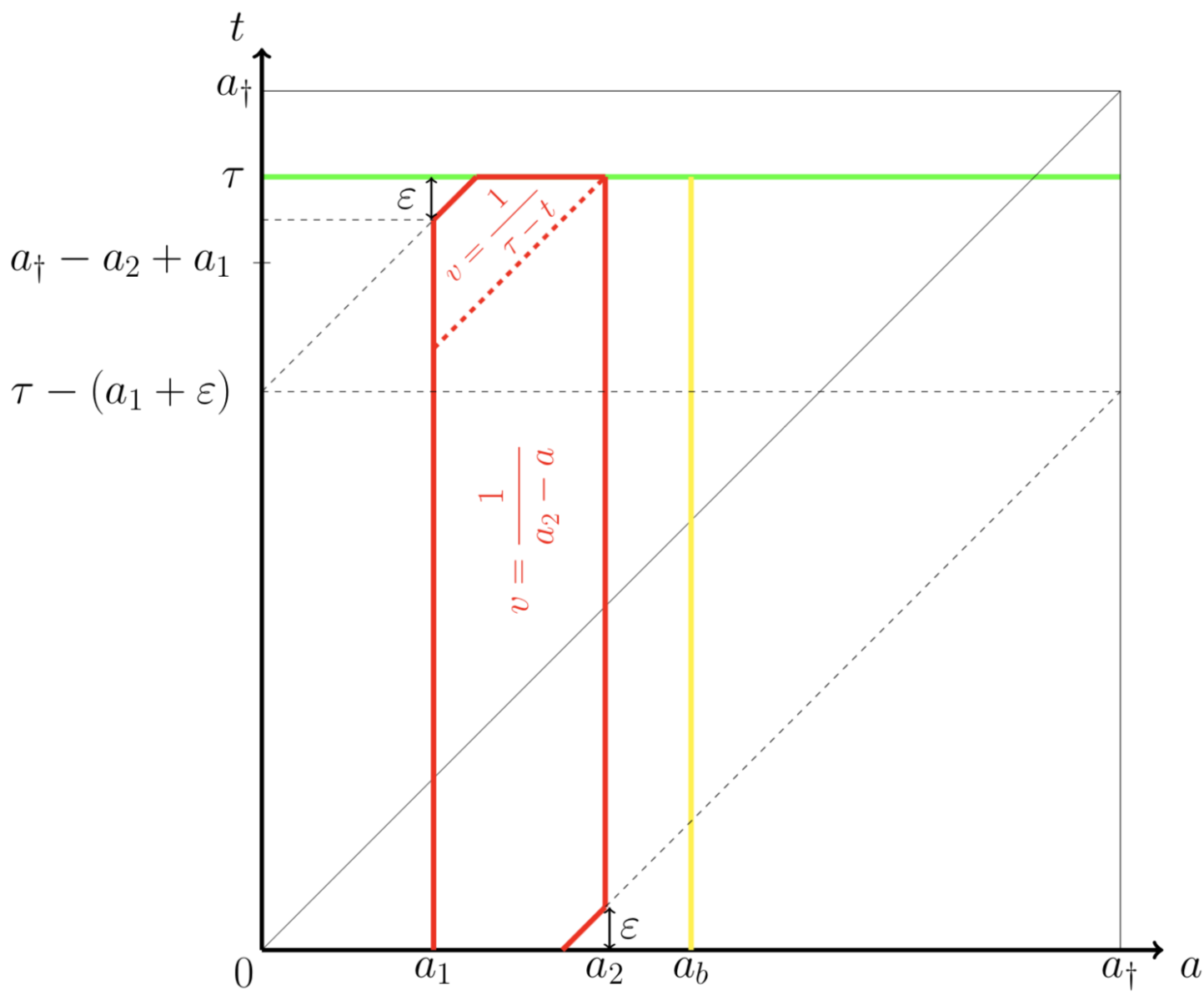

Figure 1: The structure of the control $v$ when $\tau>a_{\dagger}-a_{2}+a_{1}$ and $a_{1}<a_{2} \leqslant a_{b}$.

The structure of $v$ is given in Figure 1. The solution to (3.1) with $u=-v p$ exists, is unique and nonnegative (see Aniţa [5]), and via the comparison principle for age-dependent population dynamics (see [5, Theorem 2.1.2]) we get that

$$
0 \leqslant p(t, a) \leqslant \tilde{p}(t, a) \text { a.e. }(t, a) \in Q_{\tau},
$$

where $\tilde{p}$ is the solution to

$$
\left\{\begin{array}{lr}
\frac{\partial \tilde{p}}{\partial t}(t, a)+\frac{\partial \tilde{p}}{\partial a}(t, a)+v(t, a) \tilde{p}(t, a)=0, & (t, a) \in Q_{\tau} \\
\tilde{p}(t, 0)=\int_{a_{b}}^{a_{\dagger}} \beta_{0}(t, a) \tilde{p}(t, a) \mathrm{d} a, & t \in(0, \tau) \\
\tilde{p}(0, a)=p_{0}(a), & a \in\left(0, a_{\dagger}\right) .
\end{array}\right.
$$


Integrating along the characteristic lines, we get that the solution $\tilde{p}$ of (3.6) satisfies

$$
\tilde{p}(t, a)=\left\{\begin{array} { l } 
{ p _ { 0 } ( a - t ) \operatorname { e x p } ( - \int _ { a - t } ^ { a } \frac { \mathrm { d } s } { a _ { 2 } - s } ) } \\
{ p _ { 0 } ( a - t ) \operatorname { e x p } ( - \int _ { a _ { 1 } } ^ { a } \frac { \mathrm { d } s } { a _ { 2 } - s } ) } \\
{ \tilde { p } ( t - a , 0 ) \operatorname { e x p } ( - \int _ { a _ { 1 } } ^ { a } \frac { \mathrm { d } s } { a _ { 2 } - s } ) } \\
{ \tilde { p } ( t - a , 0 ) \operatorname { e x p } ( - \int _ { t - a + a _ { 1 } } ^ { t } \frac { \mathrm { d } s } { \tau - s } ) }
\end{array} \quad \text { if } \left\{\begin{array}{l}
\varepsilon-a_{2} \leqslant t-a \leqslant-a_{1}, \\
a \in\left[a_{1}, a_{2}\right], \\
-a_{1} \leqslant t-a \leqslant 0, \\
a \in\left[a_{1}, a_{2}\right], \\
0<t-a<\tau-a_{2}, \\
a \in\left[a_{1}, a_{2}\right], \\
\tau-a_{2} \leqslant t-a \leqslant \tau-\left(a_{1}+\varepsilon\right), \\
a \in\left[a_{1}, a_{2}\right],
\end{array}\right.\right.
$$

so that we have

$$
\tilde{p}(t, a)= \begin{cases}\frac{a_{2}-a}{a_{2}-(a-t)} p_{0}(a-t) & \text { if }\left\{\begin{array} { l } 
{ \varepsilon - a _ { 2 } \leqslant t - a \leqslant - a _ { 1 } , } \\
{ \frac { a _ { 2 } - a } { a _ { 2 } - a _ { 1 } } p _ { 0 } ( a - t ) } \\
{ \frac { a _ { 2 } - a } { a _ { 2 } - a _ { 1 } } \tilde { p } ( t - a , a _ { 2 } ] , } \\
{ - a _ { 1 } \leqslant t - a \leqslant 0 , } \\
{ a \in [ a _ { 1 } , a _ { 2 } ] , } \\
{ \frac { \tau - t } { \tau - ( t - a + a _ { 1 } ) } \tilde { p } ( t - a , 0 ) }
\end{array} \quad \text { if } \left\{\begin{array}{l}
0<-a<\tau-a_{2}, \\
a \in\left[a_{1}, a_{2}\right], \\
\tau-a_{2} \leqslant t-a \leqslant \tau-\left(a_{1}+\varepsilon\right), \\
a \in\left[a_{1}, a_{2}\right] .
\end{array}\right.\right.\end{cases}
$$

We may conclude from the above formula that we have

$$
\begin{cases}\tilde{p}\left(t, a_{2}\right)=0 & \text { if } \quad t \in(\varepsilon, \tau), \\ \tilde{p}(\tau, a)=0 & \text { if } \quad a \in\left(a_{1}+\varepsilon, a_{2}\right) .\end{cases}
$$

Integrating on the characteristic lines the equation satisfied by $\tilde{p}$, we get from (3.8) that

$$
\tilde{p}(t, a)=0 \quad \text { if } \quad(t, a) \in\left[\tau-\left(a_{1}+\varepsilon\right), \tau\right] \times\left[a_{2}, a_{\dagger}\right] .
$$

Since $a_{b} \geqslant a_{2}$, we obviously get from the above equation that

$$
\tilde{p}(t, a)=0 \quad \text { if } \quad(t, a) \in\left[\tau-\left(a_{1}+\varepsilon\right), \tau\right] \times\left[a_{b}, a_{\dagger}\right] .
$$

Since $\tilde{p}$ satisfies the renewal law, we may infer that

$$
\tilde{p}(t, 0)=0 \quad \text { if } \quad t \in\left[\tau-\left(a_{1}+\varepsilon\right), \tau\right]
$$

and integrating along the characteristic lines we get that

$$
\tilde{p}(\tau, a)=0 \quad \text { if } \quad a \in\left[0, a_{1}+\varepsilon\right] .
$$

By (3.8), (3.9) and (3.10) we may conclude that

$$
\tilde{p}(\tau, a)=0 \quad \text { a.e. } a \in\left(0, a_{\dagger}\right) .
$$

It remains to prove that the control $u=-v p$ belongs to $L^{2}\left(Q_{\tau}\right)$. For almost every $(t, a) \in Q_{\tau}$, 
from (3.4) and (3.7) we have

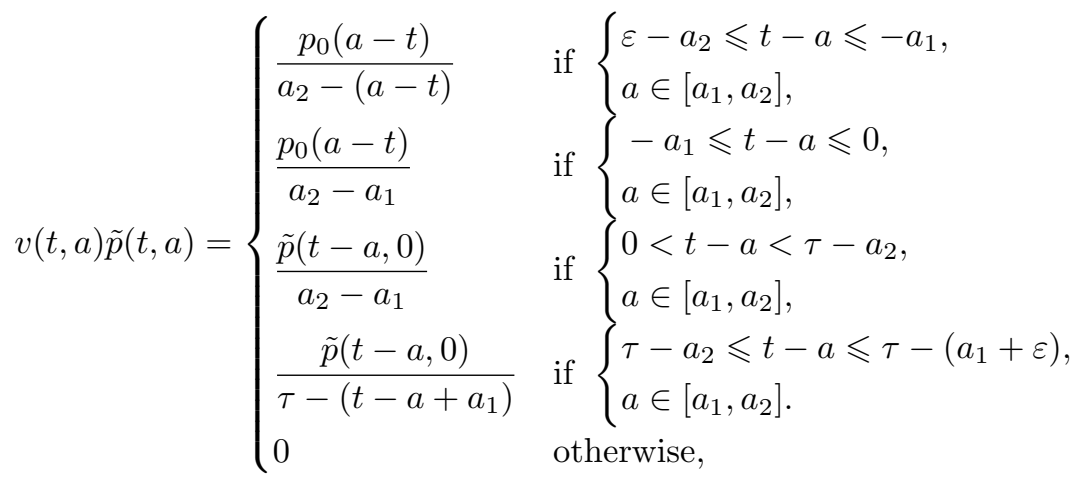

and this implies that

$$
|v(t, a) \tilde{p}(t, a)| \leqslant\left\{\begin{array}{l}
\frac{\left|p_{0}(a-t)\right|}{\varepsilon} \quad \text { if }\left\{\begin{array}{l}
\varepsilon-a_{2} \leqslant t-a \leqslant-a_{1}, \\
a \in\left[a_{1}, a_{2}\right],
\end{array}\right. \\
\frac{\left|p_{0}(a-t)\right|}{a_{2}-a_{1}} \\
\frac{|\tilde{p}(t-a, 0)|}{a_{2}-a_{1}} \\
\text { if }\left\{\begin{array} { l } 
{ a _ { 1 } \leqslant t - a \leqslant 0 , } \\
{ a \in [ a _ { 1 } , a _ { 2 } ] , } \\
{ 0 < t - a < \tau - a _ { 2 } , } \\
{ \frac { | \tilde { p } ( t - a , 0 ) | } { \varepsilon } }
\end{array} \text { if } \left\{\begin{array}{l}
\left.\tau-a_{1}, a_{2}\right], \\
a \in\left[a_{1}, a_{2}\right] .
\end{array}\right.\right. \\
\text { otherwise. }
\end{array}\right.
$$

Since $\tilde{p} \in L^{2}\left(Q_{\tau}\right)$, we may infer from the renewal law that we have $\tilde{p}(\cdot, 0) \in L^{2}(0, \tau)$, so that from (3.11) we have $v \tilde{p} \in L^{2}\left(Q_{\tau}\right)$. Given (3.5), it follows that we have $v p \in L^{2}\left(Q_{\tau}\right)$, which concludes the proof of Proposition 3.1 in the case where $a_{2} \leqslant a_{b}$.

\subsection{The second case : $\tau>a_{\dagger}-a_{2}+a_{1}$ and $a_{1}<a_{b}<a_{2}$}

This subsection is devoted to prove Proposition 3.1, in the case where $\tau>a_{\dagger}-a_{2}+a_{1}$ and $a_{1}<a_{b}<a_{2}$.

Proof of Proposition 3.1 with $a_{b}<a_{2}$. Suppose that $\tau>a_{\dagger}-a_{2}+a_{1}$ and $a_{1}<a_{b}<a_{2}$. Without loss of generality, we may assume that $\tau<a_{\dagger}$. We may also assume, without loss of generality, that we have $a_{b} \in\left(a_{1}, \tau\right)$ : indeed, in the case when there exists $a_{b}>0$ satisfying (3.3) such that $a_{b} \geqslant \tau$, we may consider $\widetilde{a_{b}}$ such that $\widetilde{a_{b}} \in\left(a_{1}, \tau\right)$ and replace $a_{b}$ by $\widetilde{a_{b}}$, noting that $\widetilde{a_{b}}$ also satisfies (3.3) since $\widetilde{a_{b}}<a_{b}$.

For any $\varepsilon>0$, denote by $\theta(\varepsilon):=\tau-\left(a_{\dagger}-a_{2}+a_{1}\right)-\varepsilon$. Let us introduce $\varepsilon>0$ small enough such that we have $a_{1}+\varepsilon<a_{b}$ and $\theta(\varepsilon)>0$ (this is possible, since $a_{1}<a_{b}$ and $\tau>a_{\dagger}-a_{2}+a_{1}$ ).

Let us prove that there exists a feedback control $u$ and a corresponding unique nonnegative solution $p$ of (3.1) satisfying

$$
p(\tau, a)=0 \quad \text { a.e. } a \in\left(0, a_{\dagger}\right) .
$$


For almost every $(t, a) \in Q_{\tau}$, let us consider the feedback control $u(t, a):=-v(t, a) p(t, a)$, where

$$
v(t, a):= \begin{cases}\frac{1}{a_{2}-a} & \text { if }\left\{\begin{array}{l}
\theta(\varepsilon)-a_{2} \leqslant t-a<\tau-a_{1}-a_{2}-\varepsilon, \\
\frac{1}{\tau-\left(a_{1}+\varepsilon\right)-t}
\end{array}\right. \\
\frac{1}{a_{b}-a} & \text { if }\left\{\begin{array}{l}
\left.a_{1}, a_{2}\right], \\
\tau-a_{1}-a_{2}-\varepsilon \leqslant t-a<\tau-a_{1}-a_{b}-\varepsilon, \\
a \in\left[a_{1}, a_{2}\right], \\
\frac{1}{\tau-t}
\end{array}\right. \\
\text { if }\left\{\begin{array}{l}
\tau-a_{1}-a_{b}-\varepsilon \leqslant t-a<\tau-a_{b}, \\
a \in\left[a_{1}, a_{b}\right],
\end{array}\right. \\
\text { if }\left\{\begin{array}{l}
\tau-a_{b} \leqslant t-a<\tau-\left(a_{1}+\varepsilon\right), \\
a \in\left[a_{1}, a_{b}\right],
\end{array}\right.\end{cases}
$$

The structure of $v$ is given in Figure 2. The solution to (3.1) with $u=-v p$ exists, is unique

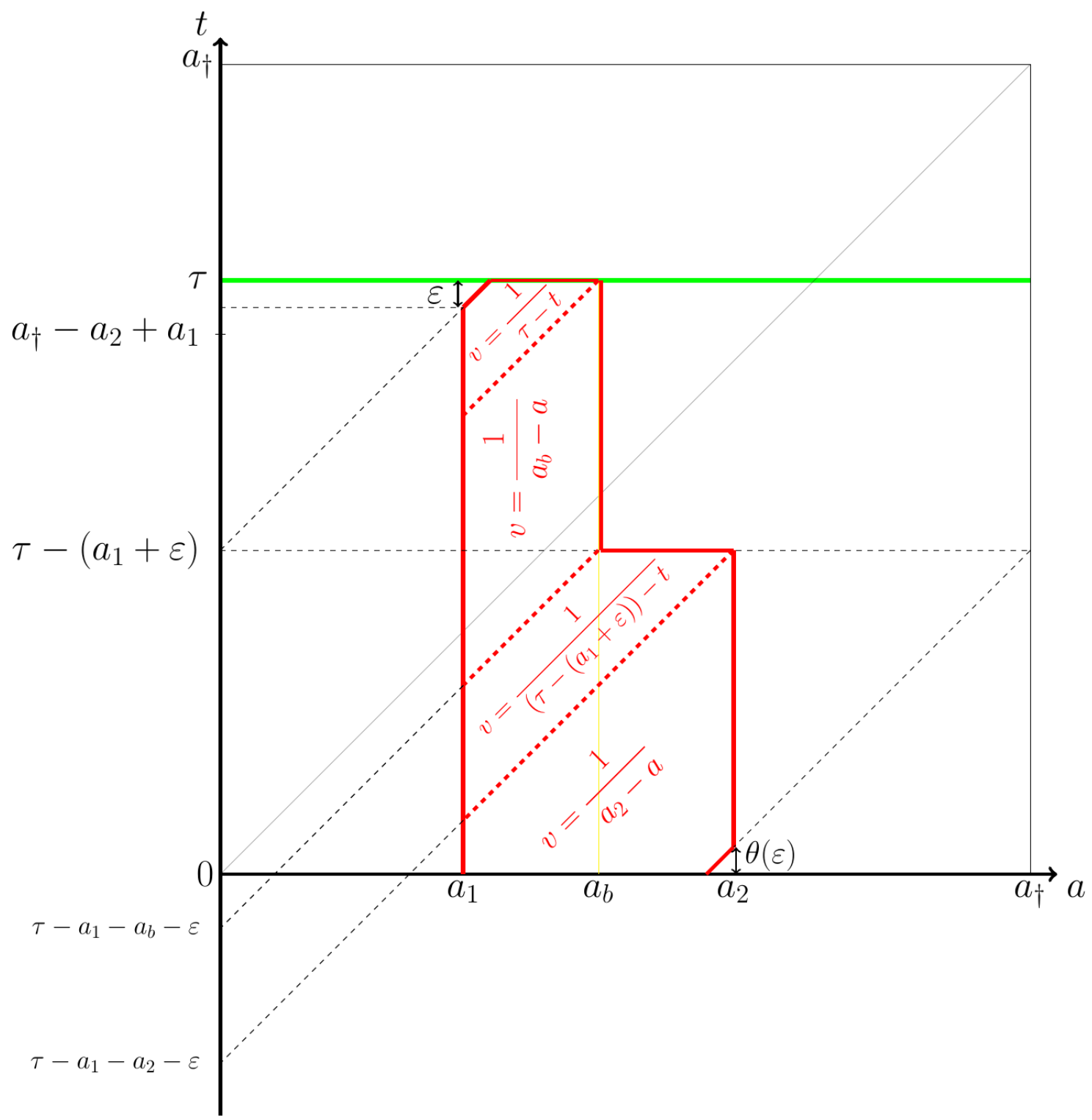

Figure 2: The structure of the control $v$ when $\tau>a_{\dagger}-a_{2}+a_{1}$ and $a_{1}<a_{b}<a_{2}$. 
and nonnegative (see Aniţa [5]), and via the comparison principle for age-dependent population dynamics (see [5, Theorem 2.1.2]) we get that

$$
0 \leqslant p(t, a) \leqslant \tilde{p}(t, a) \text { a.e. }(t, a) \in Q_{\tau},
$$

where $\tilde{p}$ is the solution to

$$
\left\{\begin{array}{lr}
\frac{\partial \tilde{p}}{\partial t}(t, a)+\frac{\partial \tilde{p}}{\partial a}(t, a)+v(t, a) \tilde{p}(t, a)=0, & (t, a) \in Q_{\tau} \\
\tilde{p}(t, 0)=\int_{a_{b}}^{a_{\dagger}} \beta_{0}(t, a) \tilde{p}(t, a) \mathrm{d} a, & t \in(0, \tau) \\
\tilde{p}(0, a)=p_{0}(a), & a \in\left(0, a_{\dagger}\right) .
\end{array}\right.
$$

We may discuss three subcases, depending on the order relationship of $\tau-a_{1}-a_{b}-\varepsilon, \tau-a_{1}-a_{2}-\varepsilon$ and 0 (since the expression of the solution $\tilde{p}$ of (3.14) may be different in each subcase). The first subcase (see below) is shown in Figure 2.

Subcase 1 : assume that $\tau-a_{1}-a_{b}-\varepsilon \leqslant 0$. Integrating along the characteristic lines, we get that the solution $\tilde{p}$ of (3.14) satisfies

$$
\tilde{p}(t, a)=p_{0}(a-t) \exp \left(-\int_{\max \left(a-t, a_{1}\right)}^{a} \frac{\mathrm{d} s}{a_{2}-s}\right)
$$

if $\theta(\varepsilon)-a_{2} \leqslant t-a<\tau-a_{1}-a_{2}-\varepsilon, a \in\left[a_{1}, a_{2}\right]$,

$$
\tilde{p}(t, a)=p_{0}(a-t) \exp \left(-\int_{\max \left(t-a+a_{1}, 0\right)}^{t} \frac{\mathrm{d} s}{\tau-\left(a_{1}+\varepsilon\right)-s}\right)
$$

if $\tau-a_{1}-a_{2}-\varepsilon \leqslant t-a<\tau-a_{1}-a_{b}-\varepsilon, a \in\left[a_{1}, a_{2}\right]$,

$$
\tilde{p}(t, a)=p_{0}(a-t) \exp \left(-\int_{\max \left(a-t, a_{1}\right)}^{a} \frac{\mathrm{d} s}{a_{b}-s}\right)
$$

if $\tau-a_{1}-a_{b}-\varepsilon \leqslant t-a \leqslant 0, a \in\left[a_{1}, a_{b}\right]$,

$$
\tilde{p}(t, a)=\tilde{p}(t-a, 0) \exp \left(-\int_{a_{1}}^{a} \frac{\mathrm{d} s}{a_{b}-s}\right)
$$

if $0<t-a \leqslant \tau-a_{b}, a \in\left[a_{1}, a_{b}\right]$,

$$
\tilde{p}(t, a)=\tilde{p}(t-a, 0) \exp \left(-\int_{t-a+a_{1}}^{t} \frac{\mathrm{d} s}{\tau-s}\right)
$$

if $\tau-a_{b}<t-a \leqslant \tau-\left(a_{1}+\varepsilon\right), a \in\left[a_{1}, a_{b}\right]$, so that we have

$$
\tilde{p}(t, a)=\frac{a_{2}-a}{a_{2}-\max \left(a-t, a_{1}\right)} p_{0}(a-t)
$$

if $\theta(\varepsilon)-a_{2} \leqslant t-a<\tau-a_{1}-a_{2}-\varepsilon, a \in\left[a_{1}, a_{2}\right]$,

$$
\tilde{p}(t, a)=\frac{\tau-\left(a_{1}+\varepsilon\right)-t}{\tau-\left(a_{1}+\varepsilon\right)-\max \left(t-a+a_{1}, 0\right)} p_{0}(a-t)
$$

if $\tau-a_{1}-a_{2}-\varepsilon \leqslant t-a<\tau-a_{1}-a_{b}-\varepsilon, a \in\left[a_{1}, a_{2}\right]$,

$$
\tilde{p}(t, a)=\frac{a_{b}-a}{a_{b}-\max \left(a-t, a_{1}\right)} p_{0}(a-t)
$$


if $\tau-a_{1}-a_{b}-\varepsilon \leqslant t-a \leqslant 0, a \in\left[a_{1}, a_{b}\right]$,

$$
\tilde{p}(t, a)=\frac{a_{b}-a}{a_{b}-a_{1}} \tilde{p}(t-a, 0)
$$

if $0<t-a \leqslant \tau-a_{b}, a \in\left[a_{1}, a_{b}\right]$,

$$
\tilde{p}(t, a)=\frac{\tau-t}{\tau-\left(t-a+a_{1}\right)} \tilde{p}(t-a, 0)
$$

if $\tau-a_{b}<t-a \leqslant \tau-\left(a_{1}+\varepsilon\right), a \in\left[a_{1}, a_{b}\right]$.

Subcase 2 : assume that $\tau-a_{1}-a_{2}-\varepsilon \leqslant 0<\tau-a_{1}-a_{b}-\varepsilon$. Integrating along the characteristic lines, we get that the solution $\tilde{p}$ of (3.14) satisfies

$$
\tilde{p}(t, a)=p_{0}(a-t) \exp \left(-\int_{\max \left(a-t, a_{1}\right)}^{a} \frac{\mathrm{d} s}{a_{2}-s}\right)
$$

if $\theta(\varepsilon)-a_{2} \leqslant t-a<\tau-a_{1}-a_{2}-\varepsilon, a \in\left[a_{1}, a_{2}\right]$,

$$
\tilde{p}(t, a)=p_{0}(a-t) \exp \left(-\int_{\max \left(t-a+a_{1}, 0\right)}^{t} \frac{\mathrm{d} s}{\tau-\left(a_{1}+\varepsilon\right)-s}\right)
$$

if $\tau-a_{1}-a_{2}-\varepsilon \leqslant t-a \leqslant 0, a \in\left[a_{1}, a_{2}\right]$,

$$
\tilde{p}(t, a)=\tilde{p}(t-a, 0) \exp \left(-\int_{t-a+a_{1}}^{t} \frac{\mathrm{d} s}{\tau-\left(a_{1}+\varepsilon\right)-s}\right)
$$

if $0<t-a \leqslant \tau-a_{1}-a_{b}-\varepsilon, a \in\left[a_{1}, a_{2}\right]$,

$$
\tilde{p}(t, a)=\tilde{p}(t-a, 0) \exp \left(-\int_{a_{1}}^{a} \frac{\mathrm{d} s}{a_{b}-s}\right)
$$

if $\tau-a_{1}-a_{b}-\varepsilon<t-a \leqslant \tau-a_{b}, a \in\left[a_{1}, a_{b}\right]$,

$$
\tilde{p}(t, a)=\tilde{p}(t-a, 0) \exp \left(-\int_{t-a+a_{1}}^{t} \frac{\mathrm{d} s}{\tau-s}\right)
$$

if $\tau-a_{b}<t-a \leqslant \tau-\left(a_{1}+\varepsilon\right), a \in\left[a_{1}, a_{b}\right]$, so that we have

$$
\tilde{p}(t, a)=\frac{a_{2}-a}{a_{2}-\max \left(a-t, a_{1}\right)} p_{0}(a-t)
$$

if $\theta(\varepsilon)-a_{2} \leqslant t-a<\tau-a_{1}-a_{2}-\varepsilon, a \in\left[a_{1}, a_{2}\right]$,

$$
\tilde{p}(t, a)=\frac{\tau-\left(a_{1}+\varepsilon\right)-t}{\tau-\left(a_{1}+\varepsilon\right)-\max \left(t-a+a_{1}, 0\right)} p_{0}(a-t)
$$

if $\tau-a_{1}-a_{2}-\varepsilon \leqslant t-a \leqslant 0, a \in\left[a_{1}, a_{2}\right]$,

$$
\tilde{p}(t, a)=\frac{\tau-\left(a_{1}+\varepsilon\right)-t}{\tau-\left(a_{1}+\varepsilon\right)-\left(t-a+a_{1}\right)} \tilde{p}(t-a, 0)
$$

if $0<t-a \leqslant \tau-a_{1}-a_{b}-\varepsilon, a \in\left[a_{1}, a_{2}\right]$,

$$
\tilde{p}(t, a)=\frac{a_{b}-a}{a_{b}-a_{1}} \tilde{p}(t-a, 0)
$$


if $\tau-a_{1}-a_{b}-\varepsilon<t-a \leqslant \tau-a_{b}, a \in\left[a_{1}, a_{b}\right]$,

$$
\tilde{p}(t, a)=\frac{\tau-t}{\tau-\left(t-a+a_{1}\right)} \tilde{p}(t-a, 0)
$$

if $\tau-a_{b}<t-a \leqslant \tau-\left(a_{1}+\varepsilon\right), a \in\left[a_{1}, a_{b}\right]$.

Subcase 3 : assume that $\tau-a_{1}-a_{2}-\varepsilon>0$. Integrating along the characteristic lines, we get that the solution $\tilde{p}$ of (3.14) satisfies

$$
\tilde{p}(t, a)=p_{0}(a-t) \exp \left(-\int_{\max \left(a-t, a_{1}\right)}^{a} \frac{\mathrm{d} s}{a_{2}-s}\right)
$$

if $\theta(\varepsilon)-a_{2} \leqslant t-a \leqslant 0, a \in\left[a_{1}, a_{2}\right]$,

$$
\tilde{p}(t, a)=\tilde{p}(t-a, 0) \exp \left(-\int_{a_{1}}^{a} \frac{\mathrm{d} s}{a_{2}-s}\right)
$$

if $0<t-a \leqslant \tau-a_{1}-a_{2}-\varepsilon, a \in\left[a_{1}, a_{2}\right]$

$$
\tilde{p}(t, a)=\tilde{p}(t-a, 0) \exp \left(-\int_{t-a+a_{1}}^{t} \frac{\mathrm{d} s}{\tau-\left(a_{1}+\varepsilon\right)-s}\right)
$$

if $\tau-a_{1}-a_{2}-\varepsilon<t-a \leqslant \tau-a_{1}-a_{b}-\varepsilon, a \in\left[a_{1}, a_{2}\right]$,

$$
\tilde{p}(t, a)=\tilde{p}(t-a, 0) \exp \left(-\int_{a_{1}}^{a} \frac{\mathrm{d} s}{a_{b}-s}\right)
$$

if $\tau-a_{1}-a_{b}-\varepsilon<t-a \leqslant \tau-a_{b}, a \in\left[a_{1}, a_{b}\right]$,

$$
\tilde{p}(t, a)=\tilde{p}(t-a, 0) \exp \left(-\int_{t-a+a_{1}}^{t} \frac{\mathrm{d} s}{\tau-s}\right)
$$

if $\tau-a_{b}<t-a \leqslant \tau-\left(a_{1}+\varepsilon\right), a \in\left[a_{1}, a_{b}\right]$, so that we have

$$
\tilde{p}(t, a)=\frac{a_{2}-a}{a_{2}-\max \left(a-t, a_{1}\right)} p_{0}(a-t)
$$

if $\theta(\varepsilon)-a_{2} \leqslant t-a \leqslant 0, a \in\left[a_{1}, a_{2}\right]$,

$$
\tilde{p}(t, a)=\frac{a_{2}-a}{a_{2}-a_{1}} \tilde{p}(t-a, 0)
$$

if $0<t-a \leqslant \tau-a_{1}-a_{2}-\varepsilon, a \in\left[a_{1}, a_{2}\right]$,

$$
\tilde{p}(t, a)=\frac{\tau-\left(a_{1}+\varepsilon\right)-t}{\tau-\left(a_{1}+\varepsilon\right)-\left(t-a+a_{1}\right)} \tilde{p}(t-a, 0)
$$

if $\tau-a_{1}-a_{2}-\varepsilon<t-a \leqslant \tau-a_{1}-a_{b}-\varepsilon, a \in\left[a_{1}, a_{2}\right]$,

$$
\tilde{p}(t, a)=\frac{a_{b}-a}{a_{b}-a_{1}} \tilde{p}(t-a, 0)
$$

if $\tau-a_{1}-a_{b}-\varepsilon<t-a \leqslant \tau-a_{b}, a \in\left[a_{1}, a_{b}\right]$,

$$
\tilde{p}(t, a)=\frac{\tau-t}{\tau-\left(t-a+a_{1}\right)} \tilde{p}(t-a, 0)
$$

if $\tau-a_{b}<t-a \leqslant \tau-\left(a_{1}+\varepsilon\right), a \in\left[a_{1}, a_{b}\right]$. 
In the three subcases, we may conclude that we have

$$
\begin{cases}\tilde{p}\left(t, a_{2}\right)=0 & \text { if } t \in\left(\theta(\varepsilon), \tau-\left(a_{1}+\varepsilon\right)\right), \\ \tilde{p}\left(\tau-\left(a_{1}+\varepsilon\right), a\right)=0 & \text { if } \quad a \in\left(a_{b}, a_{2}\right), \\ \tilde{p}\left(t, a_{b}\right)=0 & \text { if } t \in\left(\tau-\left(a_{1}+\varepsilon\right), \tau\right), \\ \tilde{p}(\tau, a)=0 & \text { if } a \in\left(a_{1}+\varepsilon, a_{b}\right) .\end{cases}
$$

Integrating on the characteristic lines the equation satisfied by $\tilde{p}$, we get that

$$
\tilde{p}(t, a)=0 \quad \text { if } \quad(t, a) \in\left[\tau-\left(a_{1}+\varepsilon\right), \tau\right] \times\left[a_{b}, a_{\dagger}\right] .
$$

Since $\tilde{p}$ satisfies the renewal law, we may infer that

$$
\tilde{p}(t, 0)=0 \quad \text { if } \quad t \in\left[\tau-\left(a_{1}+\varepsilon\right), \tau\right],
$$

and integrating along the characteristic lines we get that

$$
\tilde{p}(\tau, a)=0 \quad \text { if } \quad a \in\left[0, a_{1}+\varepsilon\right] .
$$

By (3.15), (3.16) and (3.17) we get that

$$
\tilde{p}(\tau, a)=0 \quad \text { a.e. } a \in\left(0, a_{\dagger}\right) .
$$

It remains to prove that, for each subcase, the control $u=-v p$ belongs to $L^{2}\left(Q_{\tau}\right)$. In order to avoid repetitive arguments, we prove that $u \in L^{2}\left(Q_{\tau}\right)$ only in the first subcase. In this subcase, for almost every $(t, a) \in Q_{\tau}$, from (3.12) and the corresponding expression of $\tilde{p}$ we have that

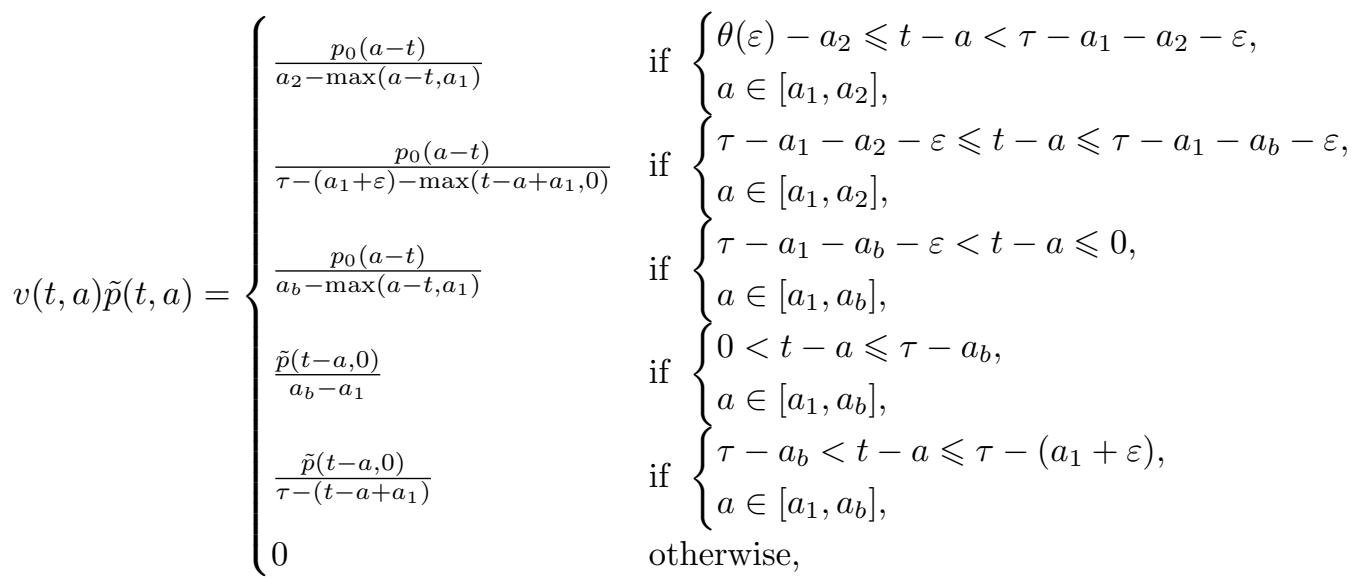

and it follows that we have

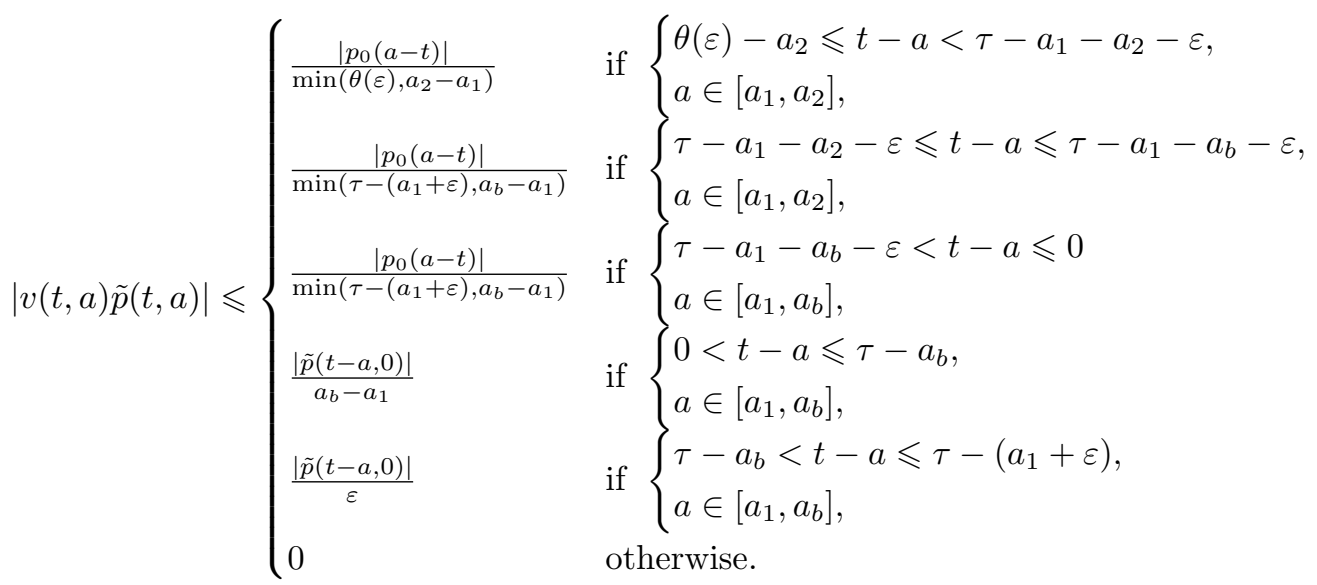

Since $\tilde{p} \in L^{2}\left(Q_{\tau}\right)$, we may infer from the renewal law that we have $\tilde{p}(\cdot, 0) \in L^{2}(0, \tau)$, so that we have $v \tilde{p} \in L^{2}\left(Q_{\tau}\right)$. Given (3.13), it follows that we have $v p \in L^{2}\left(Q_{\tau}\right)$, which concludes the proof of Proposition 3.1 in the case where $a_{2}>a_{b}$. 


\section{The linear case : negative null controllability results when the young individuals do not reproduce}

This section is devoted to prove that the results obtained in Proposition 3.1 in the linear setting are sharp, in the sense that if we do not control before the individuals start to reproduce or if the control time horizon $\tau$ is too small, then system (3.1) may not be null controllable. In this whole section, we consider the linear controlled system (3.1) subject to assumptions (L1) - (L4) stated in Section 3. We will prove the following Proposition 4.1 which is the "linear version" of Theorem 1.2:

Proposition 4.1. With the above notations and assumptions,

(i) Assume that there exists $a_{b} \in\left(0, a_{\dagger}\right)$ and $a_{m} \in\left(0, a_{\dagger}\right]$ with $a_{b}<a_{m}$ such that, for almost every $t \in(0, \tau)$ we have

$$
\beta_{0}(t, a)>0 \quad \text { a.e. } a \in\left(a_{b}, a_{m}\right)
$$

If $a_{1}>a_{b}$, then for every $\tau>0$, there exists $p_{0} \in L^{2}\left(0, a_{\dagger}\right)$ with $p_{0}(a) \geqslant 0$ a.e. $a \in\left(0, a_{\dagger}\right)$ such that there is no control $u$ and a corresponding nonnegative solution $p$ to (3.1) satisfying

$$
p(\tau, a)=0 \quad \text { a.e. } a \in\left(0, a_{\dagger}\right) .
$$

(ii) If $\tau<a_{\dagger}-a_{2}+a_{1}$, then there exists a function $\beta_{0}$ satisfying (L1) - (L2) and an initial population density $p_{0} \in L^{2}\left(0, a_{\dagger}\right)$ with $p_{0}(a) \geqslant 0$ a.e. $a \in\left(0, a_{\dagger}\right)$ such that there is no control $u$ and a corresponding nonnegative solution $p$ to (3.1) satisfying

$$
p(\tau, a)=0 \quad \text { a.e. } a \in\left(0, a_{\dagger}\right) .
$$

Proof of Proposition 4.1 - (i). Suppose that there exists $a_{b} \in\left(0, a_{\dagger}\right)$ and $a_{m} \in\left(0, a_{\dagger}\right]$ with $a_{b}<a_{m}$ such that (4.1) holds. Assume, first, that $a_{1}=a_{b}$ and let $\tau>0$. Since $a_{1}>0$, note that there exists $m \in \mathbb{N}$ and $r \in\left[0, a_{1}\right)$ such that $\tau=m a_{1}+r$. We may suppose, without loss of generality, that $m \geqslant 2$. Let $p_{0} \in L^{2}\left(0, a_{\dagger}\right)$ such that

$$
p_{0}(a)>0 \quad \text { a.e. } a \in\left(0, a_{1}-r\right) .
$$

Suppose that there exists a control function $u \in L^{2}\left(Q_{\tau}\right)$ and a corresponding nonnegative solution $p$ of (3.1) such that

$$
p(\tau, a)=0 \quad \text { a.e. } a \in\left(0, a_{\dagger}\right) .
$$

Since $p(\tau, a)=0$ for almost every $a \in\left(0, a_{1}\right)$, integrating along the characteristic lines we have that

$$
p(t, 0)=0 \quad \text { a.e. } t \in\left(\tau-a_{1}, \tau\right) .
$$

Since $p$ satisfies the renewal law, for almost every $t \in\left(\tau-a_{1}, \tau\right)$ we have that

$$
p(t, 0)=\int_{a_{b}}^{a_{\dagger}} \beta_{0}(t, a) p(t, a) \mathrm{d} a=\int_{a_{b}}^{a_{m}} \beta_{0}(t, a) p(t, a) \mathrm{d} a+\int_{a_{m}}^{a_{\dagger}} \beta_{0}(t, a) p(t, a) \mathrm{d} a .
$$

Since $p(t, a) \geqslant 0$ for almost every $(t, a) \in Q_{\tau}$, using (4.3) and (4.4) we have, for almost every $t \in\left(\tau-a_{1}, \tau\right)$,

$$
\int_{a_{b}}^{a_{m}} \beta_{0}(t, a) p(t, a) \mathrm{d} a=0 .
$$

Using the assumptions on the function $\beta_{0}$ and the fact that $p(t, a) \geqslant 0$ for almost every $(t, a) \in Q_{\tau}$, together with the above equality (4.5) we have that

$$
p(t, a)=0 \quad \text { a.e. }(t, a) \in\left(\tau-a_{1}, \tau\right) \times\left(a_{b}, a_{m}\right) .
$$




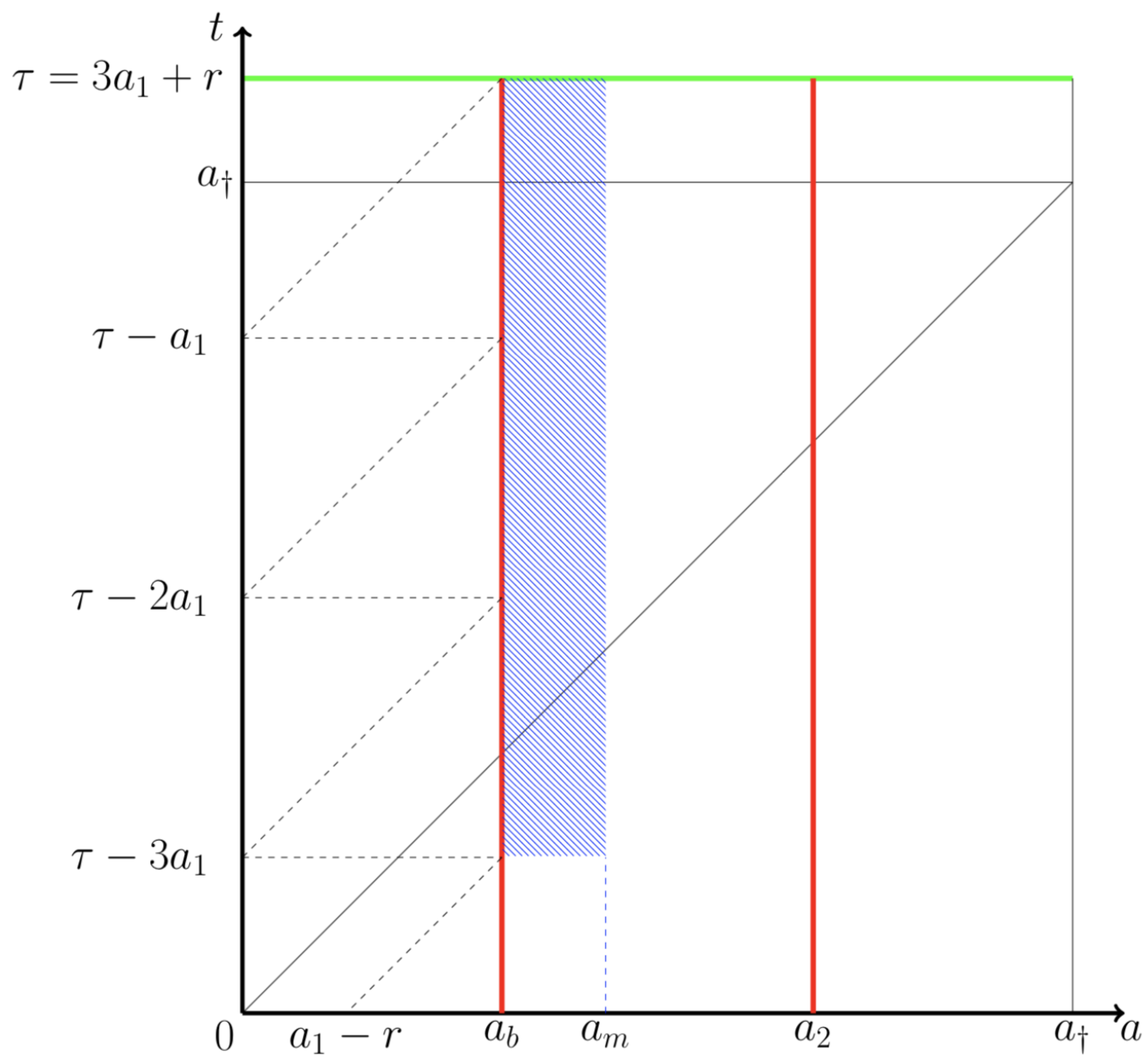

Figure 3: In this figure, we have $\tau=3 a_{1}+r$ with $r \in\left[0, a_{1}\right)$. If we suppose that the population vanishes in time $\tau$, we may deduce that the population vanishes in the whole blue dashed domain, so that the initial population has to be null in the age interval $\left(0, a_{1}-r\right)$.

Integrating along the characteristic lines we have that

$$
p(t, 0)=0 \quad \text { a.e. } t \in\left(\tau-2 a_{1}, \tau-a_{1}\right) .
$$

Using and induction argument, we may infer that

$$
p(t, 0)=0 \quad \text { a.e. } t \in\left(\tau-(m-1) a_{1}, \tau-m a_{1}\right) .
$$

Recall that $\tau-m a_{1}=r$. Using again the renewal law, it follows from the above equality that

$$
p(t, a)=0 \quad \text { a.e. }(t, a) \in\left(\tau-(m-1) a_{1}, r\right) \times\left(a_{b}, a_{m}\right),
$$

and integrating along the characteristic lines we have that

$$
p(0, a)=0 \quad \text { a.e. } a \in\left(0, a_{1}-r\right),
$$

which is a contradiction with (4.2) (see Figure 3).

Since it is not possible to bring the population to zero in any time $\tau$ in the case where $a_{1}=a_{b}$, we may infer that it is not possible to bring the population to zero in the case where $a_{1}>a_{b}$, so that Propositon 4.1 - (i) is proved. 
Proof of Proposition 4.1 - (ii). Let $\tau<a_{\dagger}-a_{2}+a_{1}$. Suppose that there exists a control function $u \in L^{2}\left(Q_{\tau}\right)$ and a corresponding nonnegative solution $p$ of (3.1) such that

$$
p(\tau, a)=0 \quad \text { a.e. } a \in\left(0, a_{\dagger}\right) .
$$

We may divide this proof in two cases.

Case 1 : suppose that $\tau<a_{\dagger}-a_{2}$. Then, for almost every $(t, a) \in Q_{\tau}$ with $a_{2} \leqslant a-t \leqslant a_{\dagger}-\tau$, we have that

$$
\int_{0}^{t} \exp \left\{-\int_{s}^{t} \mu_{0}(\sigma, a-t+\sigma) \mathrm{d} \sigma\right\} \chi_{\left[a_{1}, a_{2}\right]}(a-t+s) u(s, a-t+s) \mathrm{d} s=0,
$$

so that from (3.2), for almost every $(t, a) \in Q_{\tau}$ with $a_{2} \leqslant a-t \leqslant a_{\dagger}-\tau$ we have that

$$
p(t, a)=\exp \left\{-\int_{0}^{t} \mu_{0}(s, a-t+s) \mathrm{d} s\right\} p_{0}(a-t),
$$

and it follows from the above equality that we have that

$$
p(\tau, a)=\exp \left\{-\int_{0}^{\tau} \mu_{0}(s, a-\tau+s) \mathrm{d} s\right\} p_{0}(a-\tau) \quad \text { a.e. } a \in\left(\tau+a_{2}, a_{\dagger}\right),
$$

so that from the above equation the condition $p(\tau, \cdot)=0$ cannot be fulfilled in the case where $p_{0}(a) \neq 0$ for almost every $a \in\left(a_{2}, a_{\dagger}-\tau\right)$.

Case 2 : suppose that $\tau \geqslant a_{\dagger}-a_{2}$. Since $p(\tau, a)=0$ for almost every $a \in\left(0, a_{1}\right)$, integrating along the characteristic lines we have that

$$
p(t, 0)=0 \quad \text { a.e. } t \in\left(\tau-a_{1}, \tau\right) .
$$

Note that, since $\tau<a_{\dagger}-a_{2}+a_{1}$, we have $\tau-a_{1}<a_{\dagger}-a_{2}$ so that from (4.6) and using the fact that $a_{\dagger}-a_{2} \leqslant \tau$, we have, in particular,

$$
p(t, 0)=0 \quad \text { a.e. } t \in\left(\tau-a_{1}, a_{\dagger}-a_{2}\right) .
$$

Since $p$ satisfies the renewal law, for almost every $t \in\left(\tau-a_{1}, a_{\dagger}-a_{2}\right)$ we have that

$$
p(t, 0)=\int_{0}^{a_{\dagger}} \beta_{0}(t, a) p(t, a) \mathrm{d} a=0 .
$$

Since $\beta_{0}(t, a) \geqslant 0$ and $p(t, a) \geqslant 0$ for almost every $(t, a) \in\left(\tau-a_{1}, a_{\dagger}-a_{2}\right) \times\left(0, a_{\dagger}\right)$, we deduce from the above equality (4.7) that we have

$$
\beta_{0}(t, a) p(t, a)=0 \quad \text { a.e. }(t, a) \in\left(\tau-a_{1}, a_{\dagger}-a_{2}\right) \times\left(0, a_{\dagger}\right) .
$$

Define the triangle

$$
\mathcal{T}:=\left\{(t, a) \in Q_{\tau}, a_{2} \leqslant a-t \leqslant a_{\dagger}-\left(\tau-a_{1}\right), a \geqslant \tau-a_{1}+a_{2}, t \geqslant \tau-a_{1}\right\} .
$$

See Figure 4 for a picture of the triangle position. From (4.8) and (4.9), for almost every $(t, a) \in \mathcal{T}$ we have

$$
\beta_{0}(t, a) p(t, a)=0 .
$$

Moreover, for almost every $(t, a) \in \mathcal{T}$ we have that

$$
\int_{0}^{t} \exp \left\{-\int_{s}^{t} \mu_{0}(\sigma, a-t+\sigma) \mathrm{d} \sigma\right\} \chi_{\left[a_{1}, a_{2}\right]}(a-t+s) u(s, a-t+s) \mathrm{d} s=0,
$$




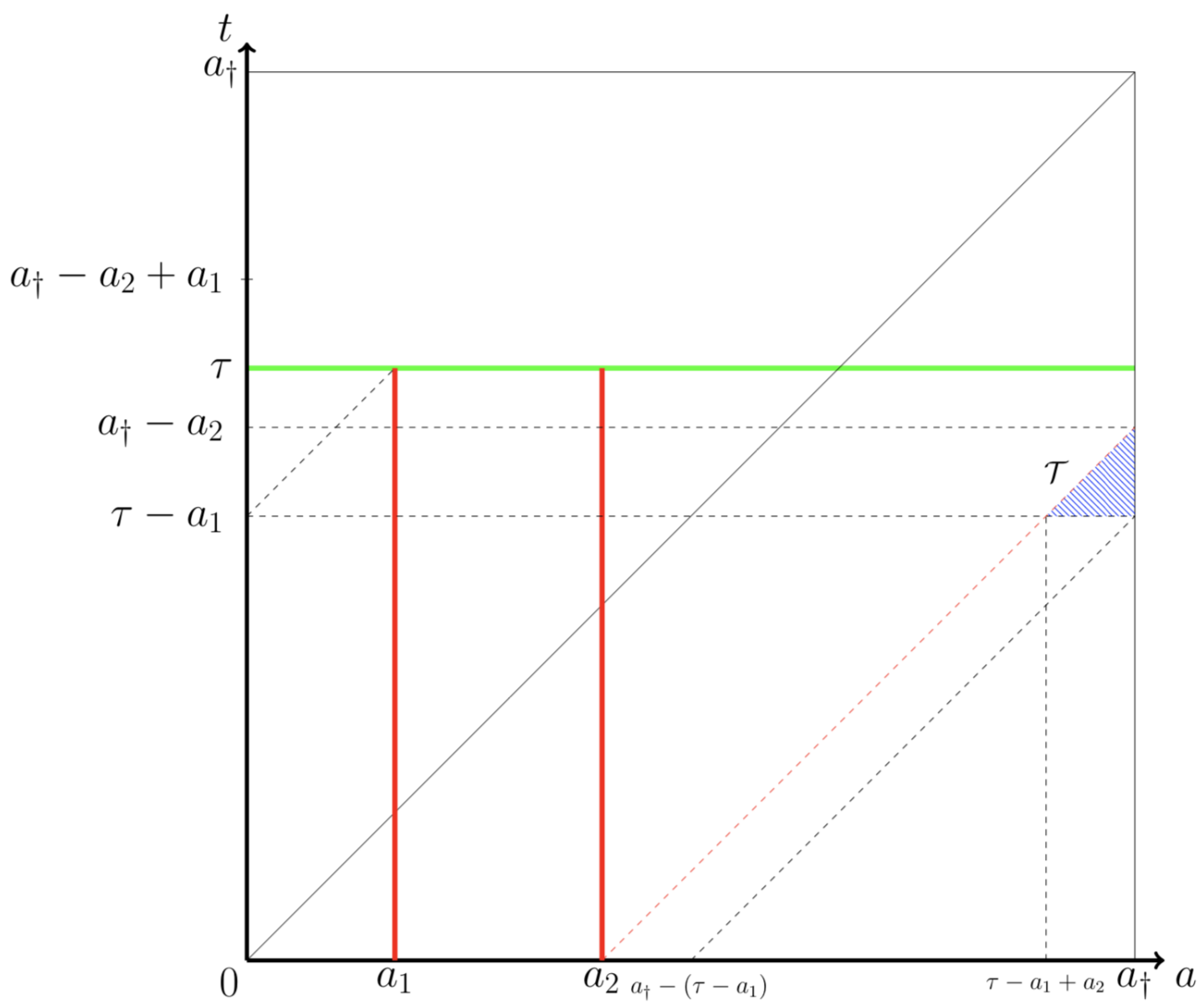

Figure 4: In this figure, we have $\tau<a_{\dagger}-a_{2}+a_{1}$ and $\tau \geqslant a_{\dagger}-a_{2}$. If we suppose that the population vanishes in time $\tau$, because of the renewal law, we may deduce that the population or the function $\beta$ has to be null within the blue dashed triangle $\mathcal{T}$ (region which is not influenced by the control). This is not the case in general : we may choose an initial condition which is strictly positive in the age interval $\left(a_{2}, a_{\dagger}-\left(\tau-a_{1}\right)\right)$ and a strictly positive function $\beta$ in the blue dashed triangle.

so that from $(3.2)$, for almost every $(t, a) \in \mathcal{T}$ we have that

$$
p(t, a)=\exp \left\{-\int_{0}^{t} \mu_{0}(s, a-t+s) \mathrm{d} s\right\} p_{0}(a-t)
$$

and using the above equality, the condition (4.10) may be rewritten as

$$
\beta_{0}(t, a) \exp \left\{-\int_{0}^{t} \mu_{0}(s, a-t+s) \mathrm{d} s\right\} p_{0}(a-t)=0 \quad \text { a.e. }(t, a) \in \mathcal{T} .
$$

This condition may not be fulfilled in general: take, for instance, $\beta_{0}(t, a)=1$ for almost every $(t, a) \in \mathcal{T}$ and $p_{0}(a)>0$ for almost every $a \in\left(a_{2}, a_{\dagger}-\left(\tau-a_{1}\right)\right)$. This concludes the proof of Proposition 4.1 - (ii).

\section{The linear case with general assumptions on the fertility rate}

This section is devoted to prove Theorem 1.3 in the linear case, when no extra assumption on the function $\beta$ is assumed (in particular, we may have $\beta>0$ in some interval of ages near zero). In 
this whole section, we consider the linear controlled system (3.1) subject to assumptions (L1) (L4) stated in Section 3. We will prove the following Proposition 5.1 which is the "linear version" of Theorem 1.3:

Proposition 5.1. With the above notations and assumptions,

(i) Assume that $a_{1}=0$ and $a_{2}>0$. If $\tau>a_{\dagger}-a_{2}$, then for every $p_{0} \in L^{2}\left(0, a_{\dagger}\right)$ with $p_{0}(a) \geqslant 0$ a.e. $a \in\left(0, a_{\dagger}\right)$, there exists $u \in L^{2}\left(Q_{\tau}\right)$ and a corresponding unique nonnegative solution $p$ of (3.1) such that

$$
p(\tau, a)=0 \quad \text { a.e. } a \in\left(0, a_{\dagger}\right) .
$$

(ii) Assume that there exists $a_{m} \in\left(0, a_{\dagger}\right]$ such that, for almost every $t \in(0, \tau)$ we have

$$
\beta_{0}(t, a)>0 \quad \text { a.e. } a \in\left(0, a_{m}\right) .
$$

If $a_{1}>0$, then for every $\tau>0$, there exists $p_{0} \in L^{2}\left(0, a_{\dagger}\right)$ with $p_{0}(a) \geqslant 0$ a.e. $a \in\left(0, a_{\dagger}\right)$ such that there is no control $u$ and a corresponding nonnegative solution $p$ to (3.1) satisfying

$$
p(\tau, a)=0 \quad \text { a.e. } a \in\left(0, a_{\dagger}\right) .
$$

(iii) If $\tau<a_{\dagger}-a_{2}$, there exists an initial population density $p_{0} \in L^{2}\left(0, a_{\dagger}\right)$ with $p_{0}(a) \geqslant 0$ a.e. $a \in\left(0, a_{\dagger}\right)$ such that there is no control $u$ and a corresponding nonnegative solution $p$ to (3.1) satisfying

$$
p(\tau, a)=0 \quad \text { a.e. } a \in\left(0, a_{\dagger}\right) .
$$

Proof of Proposition 5.1 - (i). Suppose that $\tau>a_{\dagger}-a_{2}$. Without loss of generality, we may assume that $\tau<a_{\dagger}$. Let us prove that there exists a feedback control $u$ and a corresponding unique nonnegative solution $p$ of (3.1) such that

$$
p(\tau, a)=0 \quad \text { a.e. } a \in\left(0, a_{\dagger}\right) .
$$

Denote by $\varepsilon:=\frac{1}{2}\left(\tau-\left(a_{\dagger}-a_{2}\right)\right)$. For almost every $(t, a) \in Q_{\tau}$, let us consider the feedback control $u(t, a):=-v(t, a) p(t, a)$, where

$$
v(t, a):= \begin{cases}\frac{1}{a_{2}-a} & \text { if }\left\{\begin{array}{l}
\varepsilon-a_{2} \leqslant t-a<a_{\dagger}-2 a_{2}+\varepsilon, \\
a \in\left[0, a_{2}\right], \\
\frac{1}{a_{\dagger}-a_{2}+\varepsilon-t}
\end{array}\right. \\
\text { if }\left\{\begin{array}{l}
a_{\dagger}-2 a_{2}+\varepsilon \leqslant t-a<a_{\dagger}-2 a_{2}+2 \varepsilon, \\
a \in\left[0, a_{2}\right],
\end{array}\right. \\
0 & \text { if }\left\{\begin{array}{l}
a_{\dagger}-2 a_{2}+2 \varepsilon \leqslant t-a<a_{\dagger}-a_{2}+\varepsilon, \\
t \in\left[a_{\dagger}-2 a_{2}+2 \varepsilon, a_{\dagger}-a_{2}+\varepsilon\right], \\
a \in\left[0, a_{2}-\varepsilon\right],
\end{array}\right. \\
\frac{1}{\tau-t} & \text { if }\left\{\begin{array}{l}
a_{\dagger}-a_{2}+\varepsilon \leqslant t \leqslant \tau, \\
a \in\left[0, a_{2}\right],
\end{array}\right. \\
0 & \text { otherwise. }\end{cases}
$$

The structure of $v$ is given in Figure 5. The solution to (3.1) with $u=-v p$ exists, is unique and nonnegative (see Aniţa [5]), and via the comparison principle for age-dependent population dynamics (see [5, Theorem 2.1.2]) we get that

$$
0 \leqslant p(t, a) \leqslant \tilde{p}(t, a) \text { a.e. }(t, a) \in Q_{\tau},
$$

where $\tilde{p}$ is the solution to

$$
\left\{\begin{array}{lr}
\frac{\partial \tilde{p}}{\partial t}(t, a)+\frac{\partial \tilde{p}}{\partial a}(t, a)+v(t, a) \tilde{p}(t, a)=0, & (t, a) \in Q_{\tau} \\
\tilde{p}(t, 0)=M \int_{0}^{a_{\dagger}} \tilde{p}(t, a) \mathrm{d} a, & t \in(0, \tau) \\
\tilde{p}(0, a)=p_{0}(a), & a \in\left(0, a_{\dagger}\right),
\end{array}\right.
$$




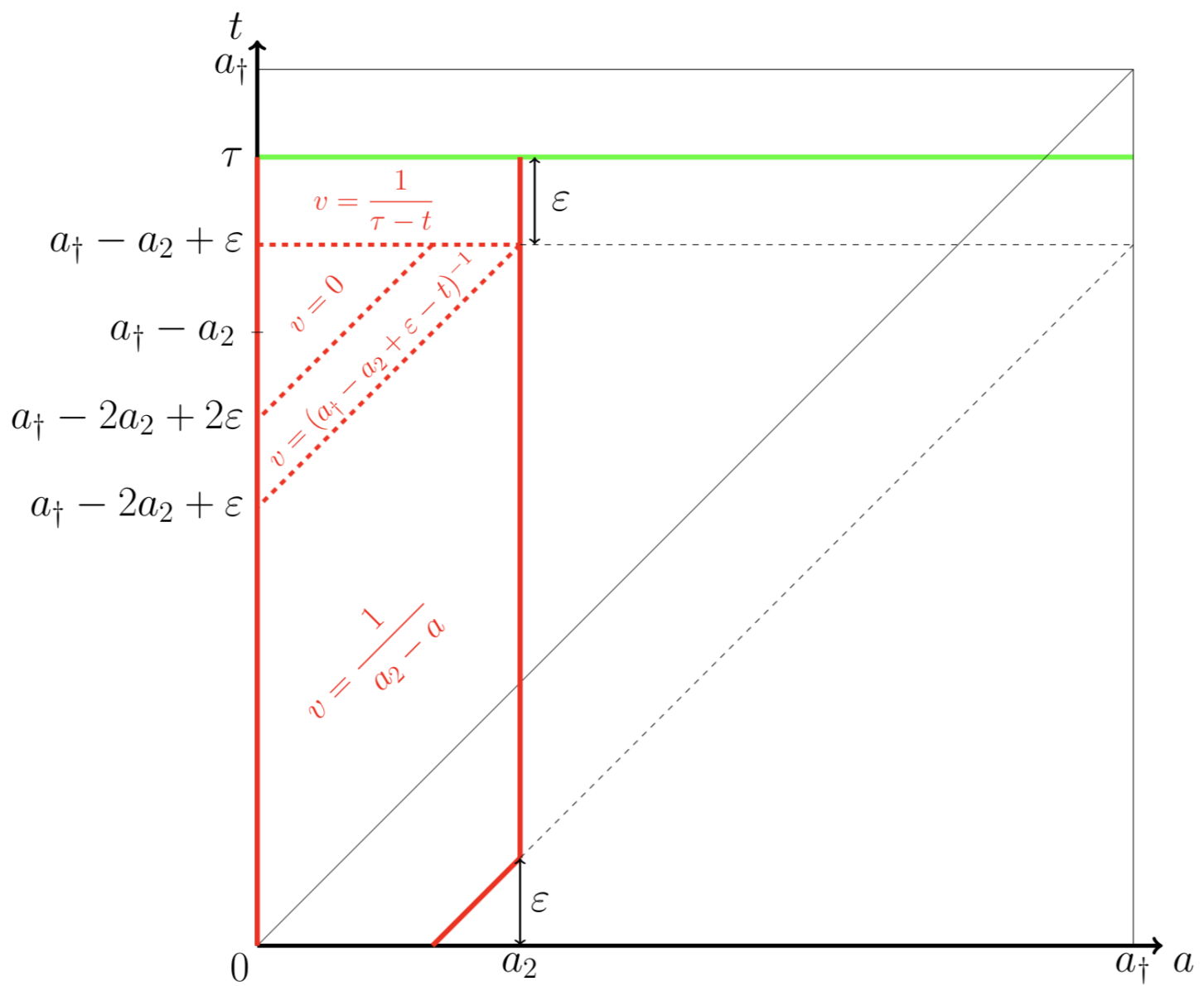

Figure 5: The structure of the control $v$ when $a_{1}=0$ and $\tau>a_{\dagger}-a_{2}$.

where $M:=\left\|\beta_{0}\right\|_{L^{\infty}\left(Q_{\tau}\right)}$.

There may be again three subcases to discuss, depending on the order relationship of $a_{\dagger}-2 a_{2}+\varepsilon$, $a_{\dagger}-2 a_{2}+2 \varepsilon$ and 0 (since the expression of the solution $\tilde{p}$ of (5.3) may be different in each subcase). Here, we do the proof only in the case when $a_{\dagger}-2 a_{2}+\varepsilon>0$ (namely, the case when $a_{2}$ is sufficiently small) which is pictured in Figure 5. In this case, integrating along the characteristic lines, we get that the solution $\tilde{p}$ of (5.3) satisfies

$$
\tilde{p}(t, a)=p_{0}(a-t) \exp \left(-\int_{a-t}^{a} \frac{\mathrm{d} s}{a_{2}-s}\right)
$$

if $\varepsilon-a_{2} \leqslant t-a \leqslant 0, a \in\left[0, a_{2}\right]$

$$
\tilde{p}(t, a)=\tilde{p}(t-a, 0) \exp \left(-\int_{0}^{a} \frac{\mathrm{d} s}{a_{2}-s}\right)
$$

if $0<t-a \leqslant a_{\dagger}-2 a_{2}+\varepsilon, a \in\left[0, a_{2}\right]$

$$
\tilde{p}(t, a)=\tilde{p}(t-a, 0) \exp \left(-\int_{t-a}^{t} \frac{\mathrm{d} s}{a_{\dagger}-a_{2}+\varepsilon-s}\right)
$$

if $a_{\dagger}-2 a_{2}+\varepsilon<t-a \leqslant a_{\dagger}-2 a_{2}+2 \varepsilon, a \in\left[0, a_{2}\right]$,

$$
\tilde{p}(t, a)=\tilde{p}(t-a, 0)
$$


if $a_{\dagger}-2 a_{2}+2 \varepsilon<t-a \leqslant a_{\dagger}-a_{2}+\varepsilon, t \in\left[a_{\dagger}-2 a_{2}+2 \varepsilon, a_{\dagger}-a_{2}+\varepsilon\right], a \in\left[0, a_{2}-\varepsilon\right]$, so that we have

$$
\tilde{p}(t, a)= \begin{cases}\frac{a_{2}-a}{a_{2}-(a-t)} p_{0}(a-t) & \text { if }\left\{\begin{array} { l } 
{ \varepsilon - a _ { 2 } \leqslant t - a \leqslant 0 , } \\
{ a \in [ 0 , a _ { 2 } ] , } \\
{ \frac { a _ { 2 } - a } { a _ { 2 } } \tilde { p } ( t - a , 0 ) } \\
{ \frac { a _ { \dagger } - a _ { 2 } + \varepsilon - t } { a _ { \dagger } - a _ { 2 } + \varepsilon - ( t - a ) } \tilde { p } ( t - a , 0 ) }
\end{array} \quad \text { if } \left\{\begin{array} { l } 
{ 0 \leqslant a _ { \dagger } - 2 a _ { 2 } + \varepsilon , } \\
{ a \in [ 0 , a _ { 2 } ] , } \\
{ a _ { \dagger } - 2 a _ { 2 } + \varepsilon < t - a \leqslant a _ { \dagger } - 2 a _ { 2 } + 2 \varepsilon , } \\
{ a \in [ 0 , a _ { 2 } ] , } \\
{ \tilde { p } ( t - a , 0 ) }
\end{array} \quad \text { if } \left\{\begin{array}{l}
a_{\dagger}-2 a_{2}+2 \varepsilon<t-a \leqslant a_{\dagger}-a_{2}+\varepsilon, \\
t \in\left[a_{\dagger}-2 a_{2}+2 \varepsilon, a_{\dagger}-a_{2}+\varepsilon\right], \\
a \in\left[0, a_{2}-\varepsilon\right] .
\end{array}\right.\right.\right.\end{cases}
$$

We may conclude from the above formulas that we have

$$
\begin{cases}\tilde{p}\left(t, a_{2}\right)=0 & \text { if } t \in\left(\varepsilon, a_{\dagger}-a_{2}+\varepsilon\right), \\ \tilde{p}\left(a_{\dagger}-a_{2}+\varepsilon, a\right)=0 & \text { if } \quad a \in\left(a_{2}-\varepsilon, a_{2}\right) .\end{cases}
$$

Integrating on the characteristic lines the equation satisfied by $\tilde{p}$, we get from (5.5) that

$$
\tilde{p}(t, a)=0 \quad \text { if } \quad(t, a) \in\left[a_{\dagger}-a_{2}+\varepsilon, \tau\right] \times\left[a_{2}, a_{\dagger}\right] .
$$

By (5.6) we get that

$$
-\frac{1}{\tau-t} \chi_{\left[0, a_{2}\right]}(a) \tilde{p}(t, a)=-\frac{1}{\tau-t} \tilde{p}(t, a)
$$

a.e. $(t, a) \in\left[a_{\dagger}-a_{2}+\varepsilon, \tau\right] \times\left(0, a_{\dagger}\right)$, and so we may infer that $\tilde{p}$ is the unique nonnegative solution to

$$
\left\{\begin{array}{lr}
\frac{\partial \tilde{p}}{\partial t}(t, a)+\frac{\partial \tilde{p}}{\partial a}(t, a)+\frac{1}{\tau-t} \tilde{p}(t, a)=0, & (t, a) \in\left(a_{\dagger}-a_{2}+\varepsilon, \tau\right) \times\left(0, a_{\dagger}\right) \\
\tilde{p}(t, 0)=M \int_{0}^{a_{\dagger}} \tilde{p}(t, a) \mathrm{d} a, & t \in\left(a_{\dagger}-a_{2}+\varepsilon, \tau\right) \\
\tilde{p}\left(a_{\dagger}-a_{2}+\varepsilon, a\right)=\tilde{p}\left(a_{\dagger}-a_{2}+\varepsilon, a\right), & a \in\left(0, a_{\dagger}\right) .
\end{array}\right.
$$

We may conclude that

$$
\tilde{p}(t, a)=h(t) g(t, a)
$$

a.e. $(t, a) \in\left[a_{\dagger}-a_{2}+\varepsilon, \tau\right] \times\left(0, a_{\dagger}\right)(\tilde{p}$ is separable $)$, where $g$ is the solution to

$$
\left\{\begin{array}{lr}
\frac{\partial g}{\partial t}(t, a)+\frac{\partial g}{\partial a}(t, a)=0, & (t, a) \in\left(a_{\dagger}-a_{2}+\varepsilon, \tau\right) \times\left(0, a_{\dagger}\right) \\
g(t, 0)=M \int_{0}^{a_{\dagger}} g(t, a) \mathrm{d} a, & t \in\left(a_{\dagger}-a_{2}+\varepsilon, \tau\right) \\
g\left(a_{\dagger}-a_{2}+\varepsilon, a\right)=\tilde{p}\left(a_{\dagger}-a_{2}+\varepsilon, a\right), & a \in\left(0, a_{\dagger}\right),
\end{array}\right.
$$

and $h$ is the solution to

$$
\left\{\begin{array}{l}
h^{\prime}(t)+\frac{1}{\tau-t} h(t)=0, \quad t \in\left(a_{\dagger}-a_{2}+\varepsilon, \tau\right) \\
h\left(a_{\dagger}-a_{2}+\varepsilon\right)=1 .
\end{array}\right.
$$

Hence for every $t \in\left[a_{\dagger}-a_{2}+\varepsilon, \tau\right]$ we have

$$
h(t)=\frac{1}{\tau-\left(a_{\dagger}-a_{2}+\varepsilon\right)}(\tau-t) .
$$


By (5.7) we get that $\tilde{p}(\tau, a)=0$ a.e. $a \in\left(0, a_{\dagger}\right)$ and consequently

$$
p(\tau, a)=0
$$

a.e. $a \in\left(0, a_{\dagger}\right)$.

It remains to prove that the control $u=-v p$ belongs to $L^{2}\left(Q_{\tau}\right)$. For almost every $(t, a) \in$ $\left(0, a_{\dagger}-a_{2}+\varepsilon\right) \times\left(0, a_{2}\right)$, from (5.1) and (5.4) we have that

$$
|v(t, a) \tilde{p}(t, a)|= \begin{cases}\frac{p_{0}(a-t)}{a_{2}-(a-t)} & \text { if }\left\{\begin{array}{l}
\varepsilon-a_{2} \leqslant t-a \leqslant 0, \\
a \in\left[0, a_{2}\right],
\end{array}\right. \\
\frac{\tilde{p}(t-a, 0)}{a_{2}} & \text { if }\left\{\begin{array}{l}
0<t-a \leqslant a_{\dagger}-2 a_{2}+\varepsilon, \\
a \in\left[0, a_{2}\right],
\end{array}\right. \\
\frac{\tilde{p}(t-a, 0)}{a_{\dagger}-a_{2}+\varepsilon-(t-a)} & \text { if }\left\{\begin{array}{l}
a_{\dagger}-2 a_{2}+\varepsilon<t-a \leqslant a_{\dagger}-2 a_{2}+2 \varepsilon, \\
a \in\left[0, a_{2}\right],
\end{array}\right. \\
0 & \text { if }\left\{\begin{array}{l}
a_{\dagger}-2 a_{2}+2 \varepsilon<t-a \leqslant a_{\dagger}-a_{2}+\varepsilon, \\
t \in\left[a_{\dagger}-2 a_{2}+2 \varepsilon, a_{\dagger}-a_{2}+\varepsilon\right], \\
a \in\left[0, a_{2}-\varepsilon\right],
\end{array}\right.\end{cases}
$$

and it follows from (5.8) that we have

$$
|v(t, a) \tilde{p}(t, a)| \leqslant \begin{cases}\frac{\left|p_{0}(a-t)\right|}{\varepsilon} & \text { if }\left\{\begin{array}{l}
\varepsilon-a_{2} \leqslant t-a \leqslant 0, \\
a \in\left[0, a_{2}\right],
\end{array}\right. \\
0<t-a \leqslant a_{\dagger}-2 a_{2}+\varepsilon, \\
\frac{|\tilde{p}(t-a, 0)|}{a_{2}} \text { if }\left\{\begin{array}{l}
a_{2}-\varepsilon \\
a \in\left[0, a_{2}\right]
\end{array}\right.\end{cases}
$$

Since $\tilde{p} \in L^{2}\left(\left(0, a_{\dagger}-a_{2}+\varepsilon\right) \times\left(0, a_{\dagger}\right)\right)$, we may infer from the renewal law that we have $\tilde{p}(\cdot, 0) \in$ $L^{2}\left(0, a_{\dagger}-a_{2}+\varepsilon\right)$, so that from (5.9) we have $v \tilde{p} \in L^{2}\left(\left(0, a_{\dagger}-a_{2}+\varepsilon\right) \times\left(0, a_{\dagger}\right)\right)$. Given (5.2), it follows that we have $v p \in L^{2}\left(\left(0, a_{\dagger}-a_{2}+\varepsilon\right) \times\left(0, a_{\dagger}\right)\right)$.

On the other hand, for almost every $(t, a) \in\left(a_{\dagger}-a_{2}+\varepsilon\right) \times\left(0, a_{2}\right)$ we have

$$
|v(t, a) p(t, a)| \leq|v(t, a) \tilde{p}(t, a)|=\frac{1}{\tau-t} h(t) g(t, a)=\frac{1}{\tau-\left(a_{\dagger}-a_{2}+\varepsilon\right)} g(t, a),
$$

and so that $v p \in L^{2}\left(\left(a_{\dagger}-a_{2}+\varepsilon, \tau\right) \times\left(0, a_{2}\right)\right)$, which ends the proof of Proposition $5.1-(i)$.

Remark 5.2. Note that actually, in the above proof, the control $u$ defined by $u(t)=-\frac{1}{\tau-t} \tilde{p}(t)$ for every $t \in\left(a_{\dagger}-a_{2}+\varepsilon, \tau\right)$ is the feedback null control as defined by (2.5) (see Section 2) associated to the Lotka-McKendrick semigroup where the functions $\beta$ and $\mu$ are defined by $\beta(a):=M$ and $\mu(a):=0$ for almost every $a \in\left(0, a_{2}\right)$.

Proof of Proposition 5.1 - (ii) and (iii). The proof of Proposition 5.1 - (ii) follows the proof of Proposition 4.1 - (i), taking $a_{b}=0$ and $a_{1}>0$, so we omit it. Moreover, the proof of Proposition 5.1 - (iii) is the same as the proof of Proposition 4.1 - (ii), Case 1. 


\section{Two applications of the linear controllability results}

In this section, we give two applications to the results obtained in Propositions 3.1, 4.1 and 5.1. Firstly, we show how to steer a population to another one (in the linear case), preserving the nonnegativity of the population. Secondly, we shall prove Theorems 1.1, 1.2 and 1.3 in the general nonlinear case, using as a main ingredient the comparison principle for age-structured populations dynamics.

\subsection{From a population to another one}

In this subsection, we give an application of Proposition 3.1 and Proposition 5.1 to a linear population control problem. The aim of this subsection is, roughly speaking, to steer an initial population density to another population density in sufficiently large time, dealing with age restriction. More precisely, let $\tau$ be a positive constant and assume that $\tilde{p}$ is solution to the system:

$$
\left\{\begin{array}{lr}
\frac{\partial \tilde{p}}{\partial t}(t, a)+\frac{\partial \tilde{p}}{\partial a}(t, a)+\mu_{0}(t, a) \tilde{p}(t, a)=\chi_{\left[a_{1}, a_{2}\right]}(a) w(t, a), & (t, a) \in Q_{\tau} \\
\tilde{p}(t, 0)=\int_{0}^{a_{\dagger}} \beta_{0}(t, a) \tilde{p}(t, a) \mathrm{d} a, & t \in(0, \tau) \\
\tilde{p}(0, a)=\tilde{p}_{0}(a), & a \in\left(0, a_{\dagger}\right),
\end{array}\right.
$$

where the functions $\mu_{0}$ and $\beta_{0}$ are assumed to follow (L1) - (L4) (see Section 3), $w \in L^{2}\left(Q_{\tau}\right)$ is a nonnegative function and $\tilde{p}_{0} \in L^{2}\left(0, a_{\dagger}\right)$ is a nonnegative initial population density. We aim to find a control function $u \in L^{2}\left(Q_{\tau}\right)$ such that the corresponding unique solution $p$ to (3.1) coincides with the solution $\tilde{p}$ to (6.1) in time $\tau$, while preserving the nonnegativity of $p$. Such a problem has been studied, for instance, in the pioneering work [6] and more recently in [8], assuming that the functions $\mu_{0}, \beta_{0}$ and $w$ do not depend on the time, the control function $u$ is supported in the age interval $\left(0, a_{0}\right)$ for some $a_{0}<a_{\dagger}$ and $\tilde{p}$ is a steady state of (3.1). The main novely we bring here is that we may be able to steer the solution $p$ of (3.1) to the solution $\tilde{p}$ of (6.1) by means of a feedback control function $u$, without considering the above mentioned restrictions. More precisely, we have

Proposition 6.1. With the above notations and assumptions, assume that at least one of the two following conditions is fulfilled:

Condition 1: there exists $a_{b} \in\left(0, a_{\dagger}\right)$ such that (3.3) is satisfied, with $a_{1}\left\langle a_{b}\right.$ and $\left.\tau\right\rangle$ $a_{\dagger}-a_{2}+a_{1}$,

Condition 2: $a_{1}=0$ and $\tau>a_{\dagger}-a_{2}$.

Suppose that $w$ and $\tilde{p}$ are both nonnegative functions in $L^{2}\left(Q_{\tau}\right)$, where $\tilde{p}$ is the solution of (6.1). Then for every $p_{0} \in L^{2}\left(0, a_{\dagger}\right)$ with $p_{0}(a) \geqslant 0$ a.e. $a \in\left(0, a_{\dagger}\right)$, there exists $u \in L^{2}\left(Q_{\tau}\right)$ and a unique nonnegative solution $p$ of (3.1) such that

$$
p(\tau, a)=\tilde{p}(\tau, a) \quad \text { a.e. } a \in\left(0, a_{\dagger}\right) .
$$

Proof. Suppose, first, that the Condition 1 of Proposition 6.1 is fulfilled. For almost every $(t, a) \in$ $Q_{\tau}$, we set

$$
u(t, a):=w(t, a)-v(t, a)(p(t, a)-\tilde{p}(t, a)) \text { a.e. }(t, a) \in Q_{\tau},
$$

where $v$ is defined by (3.4) (resp. by (3.12)) in the case where $a_{2} \leqslant a_{b}$ (resp. in the case where $a_{b}<a_{2}$ ). 
Denoting by $h:=p-\tilde{p}$, using (3.1), (6.1) together with the above definition of the control function $u$ given by (6.2), we get that $h$ is solution of the following system:

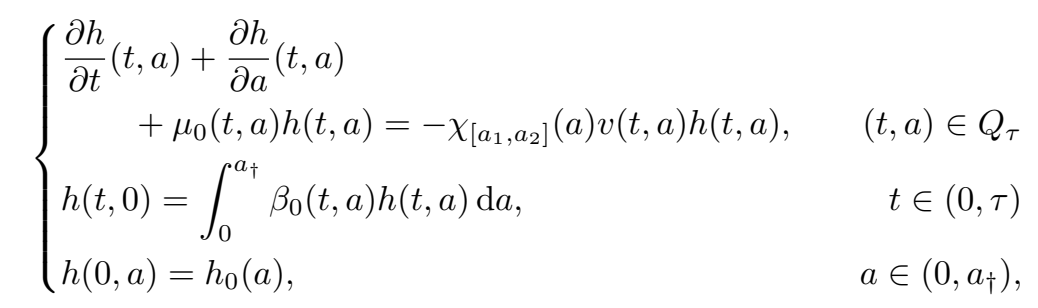

where $h_{0}(a):=p_{0}(a)-\tilde{p}_{0}(a)$ for almost every $a \in\left(0, a_{\dagger}\right)$. From the proof of Proposition 3.1, we get that the solution $h$ of $(6.3)$ satisfies $h(\tau, \cdot)=0$, with $v h \in L^{2}\left(Q_{\tau}\right)$. It follows that we have $p(\tau, \cdot)=\tilde{p}(\tau, \cdot)$ and $u=w-v h \in L^{2}\left(Q_{\tau}\right)$.

It remains to show that the unique solution $p$ to (3.1) corresponding to $u$ defined by (6.2) is nonnegative. Since $u$ is defined by (6.2), we get that $p$ is solution to

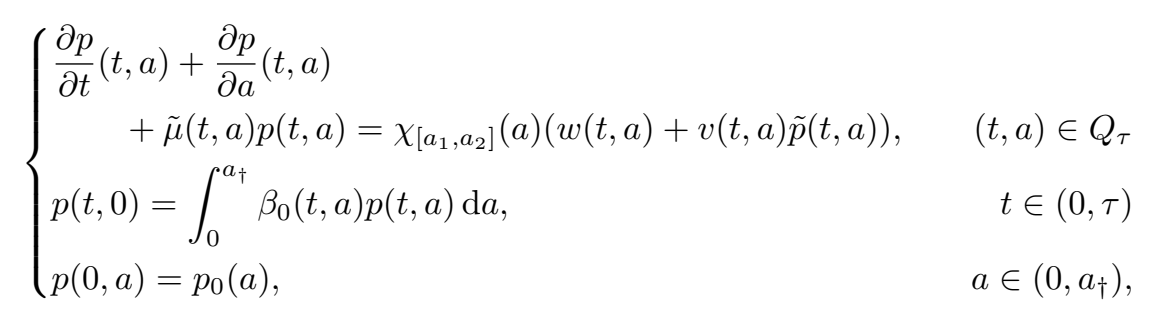

where $\tilde{\mu}(t, a):=\mu_{0}(t, a)+\chi_{\left[a_{1}, a_{2}\right]} v(t, a)$ for almost every $(t, a) \in Q_{\tau}$. Noting that, for almost every $(t, a) \in Q_{\tau}$ we have

$$
\chi_{\left[a_{1}, a_{2}\right]}(a)(w(t, a)+v(t, a) \tilde{p}(t, a)) \geqslant 0 \text { a.e. }(t, a) \in Q_{\tau},
$$

it follows from the comparison principle for linear age-structured population dynamics (see, for instance, [5]) that the solution $p$ of (6.4) satisfies $p(t, a) \geqslant 0$ for almost every $(t, a) \in Q_{\tau}$.

In the case where the Condition 2 is fulfilled, we may consider $v$ as defined by (5.1) and follow the above proof to get the desired result.

\subsection{From the linear to the nonlinear case}

In this subsection, we prove Theorems 1.1, 1.2 and 1.3 in the general case when both functions $\mu$ and $\beta$ may depend on theirs three variables. The proof of Theorem 1.1 is relied on the comparison result for age-dependent population dynamics (see, for instance, [5]) and the structure of the feedback control obtained in Section 3 relatively to the linear case.

Proof of Theorem 1.1. Assume that there exists $a_{b}>0$ such that (1.3) holds. Suppose that $a_{1}<$ $a_{b}$, and let $\tau>a_{\dagger}-a_{2}+a_{1}$. Suppose, first, that $a_{2} \leqslant a_{b}$. Denote by $v$ the function defined by (3.4). The solution $p$ to (1.1) with $u:=-v p$ exists, is unique, and via the comparison result for age-dependent population dynamics, we get that

$$
0 \leqslant p(t, a) \leqslant \bar{p}(t, a) \text { a.e. }(t, a) \in Q_{\tau},
$$

where $\bar{p}$ is solution to the linear system:

$$
\left\{\begin{array}{rrrl}
\frac{\partial \bar{p}}{\partial t}(t, a) & +\frac{\partial \bar{p}}{\partial a}(t, a) & & \\
& +\left(\mu(t, a, 0)+v(t, a) \chi_{\left[a_{1}, a_{2}\right]}(a)\right) \bar{p}(t, a)=0, & & (t, a) \in Q_{\tau} \\
\bar{p}(t, 0) & =\int_{0}^{a_{\dagger}} \beta_{+}(t, a) \bar{p}(t, a) \mathrm{d} a, & & t \in(0, \tau) \\
\bar{p}(0, a)=p_{0}(a), & & a \in\left(0, a_{\dagger}\right) .
\end{array}\right.
$$


From the proof of Proposition 3.1, we get that the solution $\bar{p}$ of $(6.6)$ satisfies $\bar{p}(\tau, \cdot)=0$, with $v \bar{p} \in L^{2}\left(Q_{\tau}\right)$, so that using (6.5) the solution $p$ to (1.1) with $u=-p v$ satisfies $p(\tau, \cdot)=0$ with $u \in L^{2}\left(Q_{\tau}\right)$.

The case where $a_{b}<a_{2}$ is similar, denoting by $v$ the function defined by (3.12). This concludes the proof of Theorem 1.1.

Let us now prove Theorem 1.2, using again the comparison principle for age-structured population dynamics and the results obtained in Section 4.

Proof of Theorem 1.2 - (i). Let $\tau>0$ and $a_{1}>0$. Assume by contradiction that there exists a control $u$ and a corresponding unique nonnegative solution $p$ of (1.1) such that $p(\tau, a)=0$ a.e. $a \in\left(0, a_{\dagger}\right)$. We have that $P \in L^{\infty}(0, \tau)$, where $P(t)=\int_{0}^{a_{\dagger}} p(t, a) d a$. Denote by $M=\|P\|_{L^{\infty}(0, \tau)}$ and by

$$
\beta_{-}(t, a):=\beta(t, a, M), \quad \mu_{+}(t, a):=\mu(t, a, 0), \quad \text { a.e. }(t, a) \in Q_{\tau} .
$$

By the comparison principle for age-structured population dynamics we get that

$$
0 \leq \tilde{p}(t, a) \leq p(t, a), \quad(t, a) \in Q_{\tau}^{1}
$$

(along almost every characteristic line), where $Q_{\tau}^{1}=(0, \tau) \times\left(0, \bar{a}_{1}\right), \bar{a}_{1}=\min \left\{a_{1}, a_{m}\right\}$, and $\tilde{p}$ is the solution to the linear system

$$
\left\{\begin{array}{lr}
\frac{\partial \tilde{p}}{\partial t}(t, a)+\frac{\partial \tilde{p}}{\partial a}(t, a)+\mu_{+}(t, a) \tilde{p}(t, a)=0, & (t, a) \in Q_{\tau}^{1} \\
\tilde{p}(t, 0)=\int_{0}^{\bar{a}_{1}} \beta_{-}(t, a) \tilde{p}(t, a) \mathrm{d} a, & t \in(0, \tau) \\
\tilde{p}(0, a)=p_{0}(a), & a \in\left(0, \bar{a}_{1}\right) .
\end{array}\right.
$$

Since $\beta_{-}(t, a)>0$ a.e. $(t, a) \in(0, \tau) \times\left(a_{b}, \bar{a}_{1}\right)$, we may conclude that for $p_{0}$ satisfying

$$
p_{0}(a)>0 \quad \text { a.e. } a \in\left(0, \bar{a}_{1}\right),
$$

we get that $\tilde{p}(t, a)>0$ along almost any characteristic line. Since we have $\tilde{p}(\tau, a)>0$ a.e. $a \in\left(0, \bar{a}_{1}\right)$, we get a contradiction with the fact that $p(\tau, a)=0$ a.e. $a \in\left(0, \bar{a}_{1}\right)$.

Proof of Theorem 1.2 - (ii). Let us take $\beta:=\beta_{0}$, where $\beta_{0}$ is chosen as at the end of the proof of Proposition 4.1 - (ii), then arguing again by contradiction and using the comparison principle for age-structured population dynamics we get the conclusion as in Theorem 1.2 - (i).

The proof of Theorem 1.3 follow the above proofs of Theorem 1.1 and 1.2, so we omit it.

\section{Lack of null controllability for the Lotka-McKendrick equation with spatial diffusion and positivity constraints}

In this section, we consider a linear controlled age-structured population model with spatial diffusion described by the following system:

$$
\left\{\begin{array}{lr}
\frac{\partial p}{\partial t}(t, a, x)+\frac{\partial p}{\partial a}(t, a, x)+\mu(t, a) p(t, a, x)-\Delta p(t, a, x) & \\
\quad=\chi_{\omega}(x) u(t, a, x), & (t, a) \in Q_{\tau}, x \in \Omega \\
\frac{\partial p}{\partial \nu}(t, a, x)=0, & (t, a) \in Q_{\tau}, x \in \partial \Omega \\
p(t, 0, x)=\int_{0}^{a_{\dagger}} \beta(t, a) p(t, a, x) \mathrm{d} a, & t \in(0, \tau), x \in \Omega \\
p(0, a, x)=p_{0}(a, x), & a \in\left(0, a_{\dagger}\right), x \in \Omega .
\end{array}\right.
$$

In the above equations: 
- $\Omega \subset \mathbb{R}^{N}, N \geqslant 1$, denotes a smooth connected bounded domain and $\Delta$ is the laplacian with respect to the variable $x$;

- $\frac{\partial}{\partial \nu}$ denotes the derivation operator in the direction of the unit outer normal to $\partial \Omega$. We thus have homogeneous Neumann boundary conditions, thus the considered population is isolated from the exterior of $\Omega$;

- $p(t, a, x)$ denotes the distribution density of the population at time $t$, of age $a$ at spatial position $x \in \Omega$;

- $p_{0}$ denotes the initial population distribution;

- $a_{\dagger} \in(0,+\infty)$ is the maximal age of an individual;

- $\beta$ and $\mu$ are nonnegative functions denoting respectively the birth and death rates;

- $\omega \subset \subset \Omega$ is a nonempty open susbet of $\Omega$ and $\chi_{\omega}$ denotes the characteristic function of $\omega$.

Let $\tau$ be a positive constant. We make the following classical assumptions on $\beta$ and $\mu$ :

(D1) $\beta \in L^{\infty}\left(Q_{\tau}\right), \beta(t, a) \geqslant 0$ for almost every $(t, a) \in Q_{\tau}$,

(D2) $\mu \in L_{l o c}^{1}\left([0, \tau] \times\left[0, a_{\dagger}\right)\right), \mu(t, a) \geqslant 0$ almost every $(t, a) \in Q_{\tau}$.

From a controllability view point, system (7.1) has been extensively studied in the past decades. The particular case when the control acts in the whole space (the case corresponding to $\omega=\Omega$ ) was investigated by Aniţa (see [5], p 148). The case when the control acts in a spatial subdomain $\omega$ was firstly studied by Ainseba [1], where the author proves the null controllability of the above system (7.1), except for a small interval of ages near zero. The case when the control acts in a spatial subdomain $\omega$ and also only for small age classes was investigated by Ainseba and Aniţa [2], for initial data $p_{0}$ in a neighborhood of the target $\widetilde{p}$. Related approximate and exact controllability issues have also been studied in Ainseba and Langlais [4], Ainseba and Iannelli, Traore [17], Kavian and Traore [12].

In a recent work, Hegoburu and Tucsnak [9] proved that, in the case where both functions $\beta$ and $\mu$ do not depend on the time variable, the above system (7.1) is null controllable in any time $\tau>0$, in the sense that for any $p_{0} \in L^{2}\left(\left(0, a_{\dagger}\right) \times \Omega\right)$, there exists a control function $u \in$ $L^{\infty}\left(0, \tau ; L^{2}\left(\left(0, a_{\dagger}\right) \times \Omega\right)\right)$ such that the corresponding solution $p$ of $(7.1)$ satisfies

$$
p(\tau, a, x)=0 \quad \text { a.e. }(a, x) \in\left(0, a_{\dagger}\right) \times \Omega .
$$

This result has been recently improved by Maity, Tucsnak and Zuazua [15], assuming that the young individuals are not able to reproduce before some age $a_{b}>0$, where the control function $u$ in system (7.1) has support in some interval of ages $\left[a_{1}, a_{2}\right]$, where $0 \leqslant a_{1}<a_{2} \leqslant a_{+}$. In [15] the authors proved the null controllability result with this additional age restriction, provided that the control time $\tau$ is large enough, and the age $a_{1}$ is smaller than $a_{b}$.

The aim of this section is to prove that, in general, the solution $p$ of the controlled system may not be positive in the whole time interval $[0, \tau]$. More precisely, we have:

Proposition 7.1. Let $\tau>0$. With the above notations and assumptions, suppose that for almost every $t \in[0, \tau]$, we have

$$
\beta(t, \cdot) \neq 0_{L^{\infty}\left(0, a_{\dagger}\right)} \quad \text { a.e. } t \geqslant 0,
$$

and denote by

$$
E_{t}:=\operatorname{ess} \operatorname{supp} \beta(t, \cdot) \quad \text { and } \quad E_{t}^{+}:=\sup E_{t} .
$$

Suppose that

$$
m:=\inf \left\{E_{t}^{+}, t \in[0, \tau]\right\}>0 .
$$


Then there exists $p_{0} \in L^{2}\left(\left(0, a_{\dagger}\right) \times \Omega\right)$ with $p_{0}(a, x) \geqslant 0$ for almost every $(a, x) \in\left(0, a_{\dagger}\right) \times \Omega$ such that there does not exists a control function $u \in L^{2}\left((0, \tau) ; L^{2}\left(\left(0, a_{\dagger}\right) \times \Omega\right)\right)$ such that

$$
p(\tau, a, x)=0 \quad \text { a.e. }(a, x) \in\left(0, a_{\dagger}\right) \times \Omega,
$$

together with

$$
p(t, a, x) \geqslant 0 \quad \text { a.e. } \quad(t, a, x) \in Q_{\tau} \times \Omega .
$$

In other words, for some well choosed nonnegative initial condition $p_{0} \in L^{2}\left(\left(0, a_{\dagger}\right) \times \Omega\right)$, it is not possible to eradicate the whole population in time $\tau$ while keeping the nonnegativity of the state trajectory.

Proof. Let $\tau>0$, and assume that (7.2) and (7.4) are satisfied. Denote by $\omega^{c}$ the complementary of $\omega$ in $\Omega$. Let $h_{0} \in L^{2}(\Omega)$ be a nonnegative function such that for almost every $x \in \omega^{c}$ we have

$$
h_{0}(x)>0 \quad \text { a.e. } x \in \omega^{c} .
$$

For almost every $(a, x) \in\left(0, a_{\dagger}\right) \times \Omega$, we set

$$
p_{0}(a, x):=h_{0}(x) .
$$

It is clear that the initial condition $p_{0}$ defined by (7.6) belongs to $L^{2}\left(\left(0, a_{\dagger}\right) \times \Omega\right)$ and is a nonnegative function. Let $\tau>0$. Suppose that there exists a control function $u \in L^{2}\left((0, \tau) ; L^{2}\left(\left(0, a_{\dagger}\right) \times \Omega\right)\right)$ such that the two following conditions are fulfilled:

$$
p(\tau, a, x)=0 \quad \text { a.e. }(a, x) \in\left(0, a_{\dagger}\right) \times \Omega,
$$

and

$$
p(t, a, x) \geqslant 0 \quad \text { a.e. } \quad(t, a, x) \in Q_{\tau} \times \Omega,
$$

where $p$ is the solution of $(7.1)$.

In the subdomain $Q_{\tau} \times \omega^{c}$, given (7.1) and (7.6), the function $p$ satisfies

$$
\left\{\begin{array}{lr}
\frac{\partial p}{\partial t}(t, a, x)+\frac{\partial p}{\partial a}(t, a, x) & \\
\quad+\mu(t, a) p(t, a, x)-\Delta p(t, a, x)=0, & (t, a) \in Q_{\tau}, x \in \omega^{c} \\
\frac{\partial p}{\partial \nu}(t, a, x)=0, & (t, a) \in Q_{\tau}, x \in \partial \Omega \\
p(t, 0, x)=\int_{0}^{a_{\dagger}} \beta(t, a) p(t, a, x) \mathrm{d} a, & t \in(0, \tau), x \in \omega^{c} \\
p(0, a, x)=h_{0}(x), & a \in\left(0, a_{\dagger}\right), x \in \omega^{c} .
\end{array}\right.
$$

By the comparison principle, for almost every $(t, a, x) \in Q_{\tau} \times \omega^{c}$, we have that

$$
p(t, a, x) \geqslant \tilde{p}(t, a, x) \geqslant 0,
$$

where the function $\tilde{p}$ satisfies the following equation:

$$
\left\{\begin{array}{lr}
\frac{\partial \tilde{p}}{\partial t}(t, a, x)+\frac{\partial \tilde{p}}{\partial a}(t, a, x) & \\
\quad+\mu(t, a) \tilde{p}(t, a, x)-\Delta \tilde{p}(t, a, x)=0, & (t, a) \in Q_{\tau}, x \in \omega^{c} \\
\tilde{p}(t, a, x)=0, & (t, a) \in Q_{\tau}, x \in \partial \omega \\
\frac{\partial \tilde{p}}{\partial \nu}(t, a, x)=0, & (t, a) \in Q_{\tau}, x \in \partial \Omega \\
\tilde{p}(t, 0, x)=\int_{0}^{a_{\dagger}} \beta(t, a) \tilde{p}(t, a, x) \mathrm{d} a, & t \in(0, \tau), x \in \omega^{c} \\
\tilde{p}(0, a, x)=h_{0}(x), & a \in\left(0, a_{\dagger}\right), x \in \omega^{c} .
\end{array}\right.
$$


Note that the above system (7.8) is separable, in the sense that the solution $\tilde{p}$ to (7.8) writes as

$$
\tilde{p}(t, a, x)=g(t, a) h(t, x) \quad \text { a.e. }(t, a, x) \in Q_{\tau} \times \omega^{c},
$$

where the functions $g \in L^{2}\left((0, \tau) ; L^{2}\left(0, a_{\dagger}\right)\right)$ and $h \in L^{2}\left((0, \tau) ; L^{2}(\Omega)\right)$ are respectively solutions to

$$
\left\{\begin{array}{lr}
\frac{\partial g}{\partial t}(t, a)+\frac{\partial g}{\partial a}(t, a)+\mu(t, a) g(t, a)=0, & (t, a) \in Q_{\tau} \\
g(t, 0)=\int_{0}^{a_{\dagger}} \beta(t, a) g(t, a) \mathrm{d} a, & t \in(0, \tau) \\
g(0, a)=1, & a \in\left(0, a_{\dagger}\right),
\end{array}\right.
$$

and

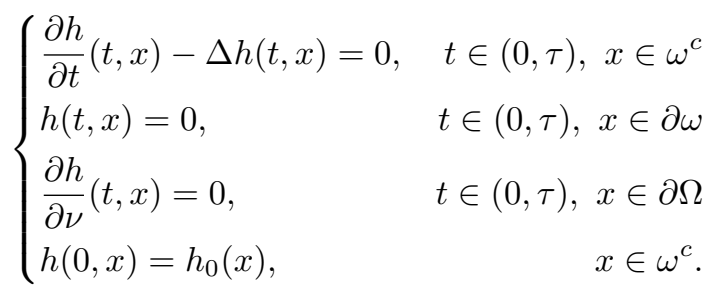

Given assumptions (7.2) and (7.4), let us prove that, for every $\tau>0$, there exists $a_{\tau} \in\left(0, a_{\dagger}\right)$ such that the solution $g$ of $(7.10)$ satisfies $g\left(\tau, a_{\tau}\right) \neq 0$. To this aim, integrating along the characteristics the equation satisfied by $g$, for almost every $(t, a) \in Q_{\tau}$ we have

$$
g(t, a)= \begin{cases}\exp \left(-\int_{0}^{t} \mu(s, a-t+s) \mathrm{d} s\right), & a \geqslant t, \\ \exp \left(-\int_{0}^{a} \mu(t-a+s, s) \mathrm{d} s\right) B(t-a), & a<t\end{cases}
$$

where

$$
B(t)=\int_{0}^{a_{\dagger}} \beta(t, a) g(t, a) \mathrm{d} a \quad \text { a.e. } t \in(0, \tau) .
$$

Suppose that there exists $\tau>0$ such that for almost every $a \in\left(0, a_{\dagger}\right)$ we have $g(t, a)=0$, where $g$ satisfies (7.10). Given (7.11), necessarily, we have $\tau \geqslant a_{\dagger}$. Assuming that $g(\tau, a)=0$ for almost every $a \in\left(0, a_{\dagger}\right)$ for some $\tau \geqslant a_{\dagger}$, from $(7.11)$ we have

$$
B(s)=0 \quad \text { a.e. } s \in\left(\tau-a_{\dagger}, \tau\right),
$$

so that, in particular, we have

$$
B\left(\tau-a_{\dagger}\right)=\int_{E_{\tau-a_{\dagger}}} \beta\left(\tau-a_{\dagger}, a\right) g\left(\tau-a_{\dagger}, a\right) \mathrm{d} a=0,
$$

where $E_{\tau-a_{\dagger}}$ is defined by (7.3). Since $\beta\left(\tau-a_{\dagger}, a\right)>0$ for almost every $a \in E_{\tau-a_{\dagger}}$ and $p$ is assumed to be positive, from (7.12) we get that for almost every $a \in E_{\tau-a_{\dagger}}$, we have

$$
g\left(\tau-a_{\dagger}, a\right)=0 \quad \text { a.e. } a \in E_{\tau-a_{\dagger}} .
$$

Denote by $E^{(1)}:=E_{\tau-a_{\dagger}}^{+}$, where $E_{\tau-a_{\dagger}}^{+}$is defined by (7.3). From (7.13), we have

$$
g\left(\tau-a_{\dagger}, E^{(1)}\right)=0 .
$$

If $E^{(1)} \geqslant \tau-a_{\dagger}$, the above condition (7.14) gives a contradiction with (7.11). Otherwise, we have $E^{(1)}<\tau-a_{\dagger}$ and integrating along the characteristics, we have

$$
g\left(\tau-a_{\dagger}-E^{(1)}, 0\right)=0 .
$$


Denote by $E^{(2)}=E_{\tau-a_{\dagger}-E^{(1)}}^{+}$. Following the previous arguments, we may deduce from the renewal law that we have

$$
g\left(\tau-a_{\dagger}-E^{(1)}, E^{(2)}\right)=0 .
$$

If $E^{(2)} \geqslant \tau-a_{\dagger}-E^{(1)}$, the above condition (7.15) gives a contradiction with (7.11). Otherwise, we have $E^{(2)}<\tau-a_{\dagger}-E^{(1)}$ and integrating along the characteristics, we have

$$
g\left(\tau-a_{\dagger}-E^{(1)}-E^{(2)}, 0\right)=0 .
$$

Define, recursively, the sequence $\left(E^{(n)}\right)_{n \geqslant 1}$ by $E^{(1)}:=E_{\tau-a_{\dagger}}^{+}$and $E^{(n+1)}:=E_{\tau-a_{\dagger}-E^{(1)}-\cdots-E^{(n)}}^{+}$. Following the above procedure, me way obtain by induction that for every $n \geqslant 1$ such that $\tau-a_{\dagger}-E^{(1)}-\cdots-E^{(n)} \geqslant 0$, we have

$$
g\left(\tau-a_{\dagger}-E^{(1)}-\cdots-E^{(n)}, E^{(n+1)}\right)=0 .
$$

Recall that there exists $m>0$ such that, for almost every $t \in[0, \tau]$ we have $E_{t}^{+} \geqslant m>0$. Thus, denote by $l$ the first integer such that

$$
\tau-a_{\dagger}-E^{(1)}-\cdots-E^{(l)}-E^{(l+1)} \leqslant 0 .
$$

Then, the condition

$$
g\left(\tau-a_{\dagger}-E^{(1)}-\cdots-E^{(l)}, E^{(l+1)}\right)=0,
$$

gives a contradiction with $(7.11)$, since $E^{(l+1)} \geqslant \tau-a_{\dagger}-E^{(1)}-\cdots-E^{(l)}$. It follows that $g(\tau, \cdot)$ cannot be the null function and there exists $a_{\tau} \in\left(0, a_{\dagger}\right)$ such that $g\left(\tau, a_{\tau}\right) \neq 0$.

Let $x \in \omega^{c}$. Suppose that $p\left(\tau, a_{\tau}, x\right)=0$, where $a_{\tau}$ is such that $g\left(\tau, a_{\tau}\right) \neq 0$. From (7.7), it follows that we have $\tilde{p}\left(\tau, a_{\tau}, x\right)=0$, so that from (7.9) we have

$$
g\left(\tau, a_{\tau}\right) h(\tau, x)=0
$$

Since $g\left(\tau, a_{\tau}\right) \neq 0$, from $(7.16)$ we have $h(\tau, x)=0$, for almost every $x \in \omega^{c}$. From the backward uniqueness for the parabolic equations, we have $h_{0}(x)=0$ for almost every $x \in \omega^{c}$. This is a contradiction with (7.5).

Remark 7.2. Using the comparison results for age-structured population dynamics with spatial diffusion and feedback controls of harvesting type it is possible to obtain null-controllability results when the control function $u$ acts in the whole habitat $\Omega$, for any $t \in(0, \tau)$ but only for some age subinterval.

\section{References}

[1] B. AinsebA, Exact and approximate controllability of the age and space population dynamics structured model, J. Math. Anal. Appl., 275 (2002), pp. 562-574.

[2] B. Ainseba And S. AniţA, Internal exact controllability of the linear population dynamics with diffusion, Electron. J. Differential Equations, (2004), pp. No. 112, 11 pp. (electronic).

[3] B. Ainseba, M. IAnnelli, et Al., Exact controllability of a nonlinear population-dynamics problem, Differential and Integral Equations, 16 (2003), pp. 1369-1384.

[4] B. Ainseba And M. Langlais, On a population dynamics control problem with age dependence and spatial structure, J. Math. Anal. Appl., 248 (2000), pp. 455-474.

[5] S. AniţA, Analysis and control of age-dependent population dynamics, vol. 11 of Mathematical Modelling: Theory and Applications, Kluwer Academic Publishers, Dordrecht, 2000. 
[6] V. Barbu, M. IAnnelli, And M. Martcheva, On the controllability of the LotkaMcKendrick model of population dynamics, J. Math. Anal. Appl., 253 (2001), pp. 142-165.

[7] M. E. Gurtin And R. C. MaCCamy, Non-linear age-dependent population dynamics, Archive for Rational Mechanics and Analysis, 54 (1974), pp. 281-300.

[8] N. Hegoburu, P. Magal, and M. Tucsnak, Controllability with positivity constraints of the Lotka-McKendrick system, SIAM J. Control Optim., 56 (2018), pp. 723-750.

[9] N. Hegoburu And M. Tucsnak, Null controllability of the Lotka-McKendrick system with spatial diffusion. working paper or preprint, Nov. 2017.

[10] M. IANnelLI, Mathematical theory of age-structured population dynamics, Giardini Editori e Stampatori in Pisa, 1995.

[11] F. KAPPel AND K. P. ZhANG, Approximation of linear age-structured population models using Legendre polynomials, J. Math. Anal. Appl., 180 (1993), pp. 518-549.

[12] O. Kavian and O. Traoré, Approximate controllability by birth control for a nonlinear population dynamics model, ESAIM Control Optim. Calc. Var., 17 (2011), pp. 1198-1213.

[13] K. Kunisch, W. Schappacher, and G. F. WebB, Nonlinear age-dependent population dynamics with random diffusion, Comput. Math. Appl., 11 (1985), pp. 155-173. Hyperbolic partial differential equations, II.

[14] D. Maity, On the Null Controllability of the Lotka-Mckendrick System. working paper or preprint, May 2018.

[15] D. Maity, M. Tucsnak, and E. Zuazua, Controllability and Positivity Constraints in Population Dynamics with Age Structuring and Diffusion. working paper or preprint, May 2018.

[16] J. Song, J. Y. Yu, X. Z. Wang, S. J. Hu, Z. X. Zhao, J. Q. Liu, D. X. Feng, and G. T. ZHU, Spectral properties of population operator and asymptotic behaviour of population semigroup, Acta Math. Sci. (English Ed.), 2 (1982), pp. 139-148.

[17] O. Traore, Null controllability of a nonlinear population dynamics problem, Int. J. Math. Math. Sci., (2006), pp. Art. ID 49279, 20.

[18] M. TuCsnak And G. Weiss, Observation and control for operator semigroups, Birkhäuser Advanced Texts: Basler Lehrbücher. [Birkhäuser Advanced Texts: Basel Textbooks], Birkhäuser Verlag, Basel, 2009.

[19] G. F. WEBB, Theory of nonlinear age-dependent population dynamics, vol. 89 of Monographs and Textbooks in Pure and Applied Mathematics, Marcel Dekker, Inc., New York, 1985. 